\title{
Modeling of multiphase mass and heat transfer in fractured high-enthalpy geothermal systems with advanced discrete fracture methodology
}

\author{
Yang Wang ${ }^{\mathrm{a}}$, Stephan de Hoop ${ }^{\mathrm{a}}$, Denis Voskov ${ }^{\mathrm{a}, \mathrm{b}, *}$, David Bruhn ${ }^{\mathrm{a}, \mathrm{c}}$, Giovanni Bertotti ${ }^{\mathrm{a}}$ \\ ${ }^{a}$ Faculty of Civil Engineering and Geosciences, Delft University of Technology, The Netherlands \\ ${ }^{\mathrm{b}}$ Department of Energy Resources Engineering, Stanford University, USA \\ ${ }^{\mathrm{c}}$ Helmholtz Center Potsdam - GFZ German Research Centre for Geosciences, Germany
}

\section{A R T I C L E I N F O}

\section{Keywords:}

High-enthalpy geothermal simulation

Thermal convection and conduction

Péclet number

Discrete-fracture model

\begin{abstract}
A B S T R A C T
Multiphase mass and heat transfer are ubiquitous in the subsurface within manifold applications. The presence of fractures over several scales and complex geometry magnifies the uncertainty of the heat transfer phenomena, which will significantly impact, or even dominate, the dynamic transport process. Capturing the details of fluid and heat transport within the fractured system is beneficial to the subsurface operations. However, accurate modeling methodologies for thermal high-enthalpy multiphase flow within fractured reservoirs are quite limited. In this work, multiphase flow in fractured geothermal reservoirs is numerically investigated. A discrete-fracture model is utilized to describe the fractured system. To characterize the thermal transport process accurately and efficiently, the resolution of discretization is necessarily optimized. A synthetic fracture model is firstly selected to run on different levels of discretization with different initial thermodynamic conditions. A comprehensive analysis is conducted to compare the convergence and computational efficiency of simulations. The numerical scheme is implemented within the Delft Advanced Research Terra Simulator (DARTS), which can provide fast and robust simulation to energy applications in the subsurface. Based on the converged numerical solutions, a thermal Péclet number is defined to characterize the interplay between thermal convection and conduction, which are the two governing mechanisms in geothermal development. Different heat transfer stages are recognized on the Péclet curve in conjunction with production regimes of the synthetic fractured reservoir. A fracture network, sketched and scaled up from a digital map of a realistic outcrop, is then utilized to perform a sensitivity analysis of the key parameters influencing the heat and mass transfer. Thermal propagation and Péclet number are found to be sensitive to flow rate and thermal parameters (e.g., rock heat conductivity and heat capacity). This paper presents a numerical simulation framework for fractured geothermal reservoirs, which provides the necessary procedures for practical investigations regarding geothermal developments with uncertainties.
\end{abstract}

\section{Introduction}

Convective and diffusive (or conductive) flow is common in the subsurface and can greatly influence the mass and heat transport process. The synergy of thermal convection and conduction plays a critical role in the development of geothermal reservoirs, where the heat is extracted with continuous injection and circulation of the heat carrier (e.g., water or $\mathrm{CO}_{2}$ (Randolph and Saar, 2018)) in manifold ways (e.g., well doublet (Willems et al., 2017), borehole heat exchanger (Hein et al., 2016), etc.). Following the cold water injection, thermal convection and conduction jointly govern the heat propagation in the geothermal reservoirs (Pruess, 1983; 1990; O'Sullivan et al., 2010). Therefore, the quantitative analysis of the interplay between convection and conduction in complex geological structures (e.g., with the presence of fractures) is not only of theoretical interest but also of practical significance.

Among the geothermal resources (Limberger et al., 2018), highenthalpy geothermal fields are given special attention owing to their substantial energy potential (Aravena et al., 2016; Kivanc Ates and Serpen, 2016; Serpen et al., 2009; Simpson and Bignall, 2016). For a highenthalpy geothermal system, either single-phase (vapor) or a two-phase mixture (vapor and liquid) can be present at the reservoir condition. The Geysers field (Geysers, 2019), an epitome of high-enthalpy geothermal systems, is the worlds largest exploited geothermal complex, producing hot steam from more than 350 wells to generate electricity in 22 power plants. The development of geothermal systems can be assisted by the inherent or induced fracture networks, especially for reservoirs with a low permeable matrix (Wang et al., 2019c). Due to their high conductivity, open fractures behave as preferential flow channels for the injected

\footnotetext{
* Corresponding author.

E-mail address: D.V.Voskov@tudelft.nl (D. Voskov).
} 
cold water. Most high-enthalpy geothermal systems either contain naturally developed fractures or require induced fractures for fluid flow.

To accurately simulate mass and heat transport in fractured geothermal systems, a suitable fracture model is critical to capture the reservoir response. Two approaches are commonly used in the representation of fractured reservoir systems (Berre et al., 2019). One is based on the continuum model, of which the typical examples are the dual-porosity model proposed by Warren and Root (1963) and the dual-permeability model presented by Gerke and van Genuchten (1993a,b). The continuum model is an efficient simplification of the fracture system but is not accurate enough when dealing with discontinuous fractures and largescale fractures dominating fluid flow (Jiang and Younis, 2015).

Another approach is the discrete-fracture model (DFM), where the fracture networks are explicitly characterized by individual control volumes. Karimi-Fard et al. (2004) proposed the DFM method, which is suitable for general-purpose reservoir simulators. This approach captures the pressure response generated by flow in fractured networks in a robust and accurate manner (Flemisch et al., 2018; Berre et al., 2021; Glser et al., 2017; Nissen et al., 2018). However, it is computationally more expensive due to the introduction of additional degrees of freedom in the computational domain. Another approach proposed by Lee et al. (2000) entails an effective representation of fractures in a computational domain and called embedded discrete-fracture model (EDFM). This approach has been greatly extended recently using multiscale techniques by Hajibeygi et al. (2011) and helps to control the computational performance and accuracy. However, the EDFM may not be as accurate as the DFM in some practical situations ()(Li and Voskov, 2021).

While operating a high-enthalpy geothermal reservoir with cold water re-injection, complicated phase behavior (water vaporization and steam condensation) will take place at the thermal front (Pruess et al., 1987). The multiphase flow and transport with complex phase changes that appear in high-enthalpy geothermal systems introduce significant challenges for numerical simulations (Coats, 1980). Several advanced techniques were proposed recently to overcome these challenges (Wong et al., 2018; Wang et al., 2019b).

In geothermal simulations, the mass and energy formulations are often tightly coupled because of the fluid thermodynamic properties (Coats et al., 1974; Chen et al., 2019). The fully-coupled fully-implicit approach is generally adopted to solve the system of equations in a robust way. During a numerical simulation, the governing equations need to be discretized in both space and time to get approximate solutions. Usually, the formulation in discretized form is nonlinear and should be linearized to facilitate solving iteratively. A Newton-Raphson based process is generally adopted to linearize the discretized formulation, which requires the values of fluid properties and their derivatives.

When complex physics (e.g., multiphase compositional flow with complex chemical reactions) is required in a model, a multiphase flash calculation is often necessary for the evaluation of accurate fluid/rock properties during each Newton iteration in the molar formulation (Collins et al., 1992; Voskov and Tchelepi, 2012). Therefore, the linearization of nonlinear equations becomes a complex problem that can take up a large portion of the overall simulation time. Voskov (2017) proposed the operator-based linearization (OBL) approach to simplify this procedure and accelerate the linearization process. Like discretization in space and time, the main idea of OBL is to discretize the physics within the space of nonlinear unknowns. The OBL approach has been utilized in the recently introduced Delft Advanced Research Terra Simulator (DARTS, 2019). The DARTS framework for geothermal modeling (Khait and Voskov, 2018b; Wang et al., 2019a; 2020b) has been validated against the state-of-the-art numerical simulators, TOUGH2 (Pruess et al., 1999) and AD-GPRS (Garipov et al., 2018), which have been widely used in geothermal energy and verified against experimental and pilot test results. Moreover, all correlations used in our code are based on the robust industrial Equation of State
(Kretzschmar and Wagner, 2019) and experimental work (Saeid et al., 2014).

In this study, we present a computational framework to numerically simulate multiphase mass and heat transport within fractured highenthalpy geothermal systems. The DFM model is used to characterize the reservoir. All simulations are run in DARTS with the OBL approach to linearize the physics. In the following description, we first introduce the adopted nonlinear formulation and the basics of the OBL approach. Then, a comprehensive study on grid optimization and heat transfer dynamics is conducted within a synthetic fracture model. Next, a realistic model based on practical outcrop measurements is utilized to perform numerical experiments with different parameters, and different scenarios are discussed.

\section{Methodology}

Mass and heat transfer in a fractured reservoir involves a thermal multiphase flow system, which requires a set of equations to depict the flow dynamics. In this section, we introduce the governing equations and detailed spatial and temporal discretization and linearization procedures. Further details related to the DARTS implementation can be found in Khait (2019).

\subsection{Governing equations}

For the investigated domain with volume $\Omega$, bounded by surface $\Gamma$, the mass and energy conservation can be expressed in a uniformly integral way, as

$\frac{\partial}{\partial t} \int_{\Omega} \boldsymbol{M}^{k} d \Omega+\int_{\Gamma} \boldsymbol{F}^{k} \cdot \boldsymbol{n} d \Gamma=\int_{\Omega} q^{k} d \Omega$.

Here, $M^{k}$ denotes the accumulation term for the $k^{\text {th }}$ component ( $k=1,2, \ldots, n$, indexing for the mass components, [e.g., water, $\mathrm{CO}_{2}$ ] and $k=n+1$ for the energy quantity). $\boldsymbol{F}^{k}$ refers to the flux term of the $k^{\text {th }}$ component. $n$ refers to the unit normal pointing outward to the domain boundary. $q^{k}$ denotes the source/sink term of the $k^{\text {th }}$ component.

The mass accumulation term collects each component distribution over $n_{p}$ fluid phases in a summation form,

$M^{k}=\phi \sum_{p=1}^{n_{p}} x_{p}^{k} \rho_{p} s_{p}, \quad k=1, \ldots, n_{c}$,

where $\phi$ is porosity, $s_{p}$ is phase saturation and $\rho_{p}$ is phase density $\left[\mathrm{kg} / \mathrm{m}^{3}\right]$ and $x_{p}^{k}$ is molar fraction of $k$ component in $p$ phase.

The energy accumulation term contains the internal energy of fluid and rock,

$M^{n_{c}+1}=\phi \sum_{p=1}^{n_{p}} s_{p} U_{p}+(1-\phi) U_{r}$.

where $U_{p}$ is fluid phase internal energy per unit volume $\left[\mathrm{kJ} / \mathrm{m}^{3}\right]$ and $U_{r}$ is rock internal energy per unit volume $\left[\mathrm{kJ} / \mathrm{m}^{3}\right]$.

We assume that the rock is compressible and represented by the change of porosity through:

$\phi=\phi_{0}\left(1+c_{r}\left(p-p_{\text {ref }}\right)\right)$,

where $\phi_{0}$ is the initial porosity, $c_{r}$ is the rock compressibility [1/bars] and $p_{\text {ref }}$ is the reference pressure [bars].

The mass flux of each component is represented by the summation over $n_{p}$ fluid phases,

$\boldsymbol{F}^{k}=\sum_{p=1}^{n_{p}} x_{p}^{k} \rho_{p} \boldsymbol{u}_{p}, \quad k=1, \ldots, n_{c}$.

Here the velocity follows the extension of Darcy's law to multiphase flow,

$u_{p}=\mathbf{K} \frac{k_{r p}}{\mu_{p}}\left(\nabla p_{p}-\gamma_{p} \nabla D\right)$ 


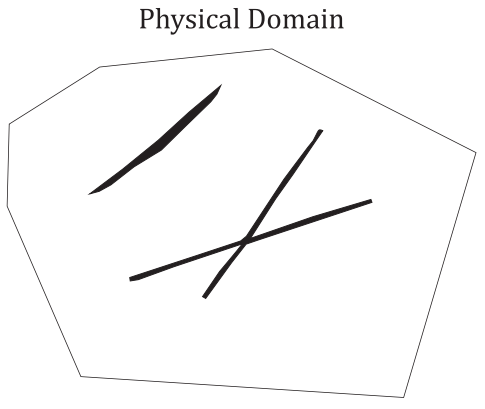

(a)

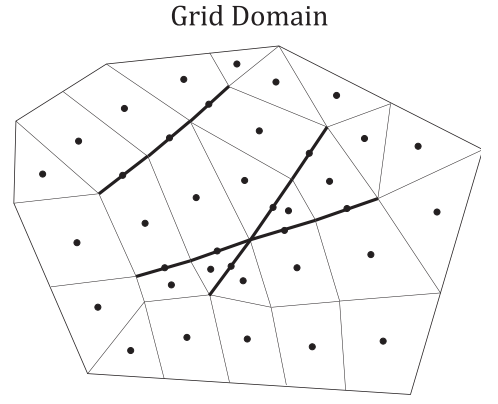

(b)

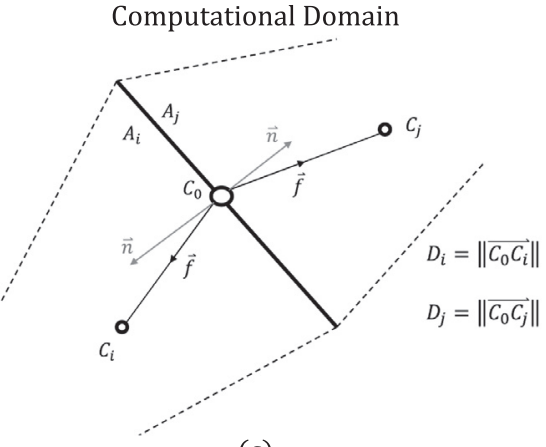

(c)

Fig. 1. Schematic representation of the DFM model (modified from Awadalla and Voskov (2018)).

where $\mathbf{K}$ is the full permeability tensor (generally zero non-diagonal elements are taken) [mD], $k_{r p}$ is the relative permeability of phase $p, \mu_{p}$ is the viscosity of phase $p[\mathrm{~Pa} \cdot \mathrm{s}], p_{p}$ is the pressure of phase $p$ [bars], $\gamma_{p}=\rho_{p} g$ is the specific weight $\left[\mathrm{N} / \mathrm{m}^{3}\right]$ and $D$ is the depth [m]. The energy flux includes the thermal convection and conduction terms,

$\boldsymbol{F}^{n_{c}+1}=\sum_{p=1}^{n_{p}} h_{p} \rho_{p} \boldsymbol{u}_{p}+\kappa \nabla T$,

where $h_{p}$ is phase enthalpy $[\mathrm{kJ} / \mathrm{kg}]$ and $\kappa$ is thermal conductivity $[\mathrm{kJ} / \mathrm{m} /$ day $/ \mathrm{K}]$.

The nonlinear equations are discretized with the finite volume method using the two-point flux approximation on general unstructured mesh in space and with the backward Euler approximation in time. For the $i^{\text {th }}$ reservoir block, the governing equation in discretized residual form reads:

$R_{i}^{k}=V_{i}\left(M_{i}^{k}\left(\omega_{i}\right)-M_{i}^{k, n}\left(\omega_{i}^{n}\right)\right)-\Delta t\left(\sum_{l} a_{l} F_{l}^{k}(\omega)+Q_{i}^{k}(\omega)\right)=0$.

Here $\omega_{i}$ refers to state variables at the current time step, $\omega_{i}^{n}$ referes to state variables at previous time step, $a_{l}$ is the contact area between neighboring grids, and $Q_{i}^{k}$ is the source or sink term of the $k^{\text {th }}$ component. In the following description, only one (water) component is assumed existing in subsurface therefore $x_{p}^{k} \equiv 1$.

\subsection{Transmissibility evaluation}

The capability of discretizing complex fractured reservoirs in DARTS has been extended with the DFM (Karimi-Fard et al., 2004). This model represents the fracture geometry in the grid domain explicitly as a lower-dimensional feature, specifically a 3D model contains 2D fractures (planes) while a 2D model contains 1D fractures (lines). This is depicted in Fig. 1 for a 2D example. To calculate transmissibilities, each fracture is assigned a specific aperture, therefore, it will have a certain volume in the computational domain.

The transmissibility between neighboring grid blocks is expressed as:

$T_{12}=\frac{\alpha_{1} \alpha_{2}}{\alpha_{1}+\alpha_{2}}$ with $\alpha_{i}=\frac{A_{i} k_{i}}{D_{i}} \mathbf{n}_{\mathbf{i}} \cdot \mathbf{f}_{\mathbf{i}}$,

where $A_{i}$ is the contact area between two neighboring blocks, $k_{i}$ is the permeability of grid block i, $D_{i}$ is the distance between the centroid of the interface and the centroid of the grid block $i, \mathbf{n}_{\mathbf{i}}$ is the unit normal of the interface pointing inside block $i, \mathbf{f}_{\mathbf{i}}$ is the unit vector along the direction of the line joining the grid block $i$ to the centroid of the interface. This equation holds true for matrix-matrix, fracture-matrix, and fracture-fracture connections. When more than two fractures intersect in the same point (2D) or line (3D), a star-delta transformation is used to calculate the transmissibility between each of the intersecting fractures. See Karimi-Fard et al. (2004) for more details.

Fracture networks usually contain complex fracture intersections that result in difficult meshing requirements. The generated mesh, therefore, often contains artifacts that negatively impact the performance of the reservoir simulation (i.e., convergence problems and numerical inaccuracies due to non-uniformity of the control volumes and large angles between $\mathbf{f}_{\mathbf{i}}$ and $\mathbf{n}_{\mathbf{i}}$ ). This is solved by using a pre-processing step in which we sequentially discretize each fracture, using the desired discretization scale (predefined size of the segment).

For fracture pre-processing, we adapted a procedure suggested by Karimi-Fard and Durlofsky (2016). During the sequential procedure, we check at every iteration if the newly placed discretized fracture segment conflicts with any previously added segment. A conflict is defined here as two fracture nodes (end-points of each discretized fracture segment) that fall within a certain radius (half the discretization accuracy) of each other. The conflicting fracture nodes will be merged and represented by one node. This pre-processing step is computationally insignificant with respect to the main fluid-flow simulation time while still greatly improving the simulation results (accuracy and computational time). This method also allows for a fast and reliable way of creating the discretized fracture network at any desired resolution while maintaining the main characteristics of the original fracture characterization.

\subsection{Operator-based linearization $(O B L)$}

The molar formulation (Faust and Mercer, 1979; Wong et al., 2015) is taken as the system nonlinear formulation, where pressure and enthalpy are chosen as the primary variables. The Newton-Raphson method is usually adopted to linearize the nonlinear equations in conventional reservoir simulation. The resulting linear system of equations on each nonlinear iteration can be expressed in the following form:

$\boldsymbol{J}\left(\boldsymbol{\omega}^{k}\right)\left(\boldsymbol{\omega}^{k+1}-\boldsymbol{\omega}^{k}\right)=-\boldsymbol{r}\left(\boldsymbol{\omega}^{k}\right)$,

where $\boldsymbol{J}\left(\boldsymbol{\omega}^{k}\right)$ is the Jacobian matrix defined at the $k^{\text {th }}$ nonlinear iteration, $\omega^{k}$ and $\omega^{k+1}$ represent the physical state (or nonlinear unknowns) at the $k^{\text {th }}$ and $k+1^{\text {th }}$ iteration.

The conventional linearization approach involves the Jacobian assembly with an accurate evaluation of property values and their derivatives to the nonlinear unknowns. The properties and their derivatives are usually directly based on either piece-wise approximations (e.g. some fluid properties) or solutions of nonlinear system (e.g. multiphase flash) with partial derivatives reconstructed using the chain rule and inverse theorem (Voskov and Tchelepi, 2012). Due to the complex combination of these properties in the governing equations, the nonlinear solver has to perform extra iterations to capture small variations in solution because of the mixed property representation.

Here, the OBL approach is utilized to improve the nonlinear behavior. For the OBL approach, the physical properties in mass and energy governing equations are agglomerated into state-dependent operators (Voskov, 2017; Khait and Voskov, 2018b). Pressure and enthalpy are taken as the unified state variables for a given control volume. Fluxrelated fluid properties are defined by the physical state of the upstream block, determined at interface $l$. The discretized mass conser- 
vation equation in operator form reads as:

$\phi_{0} V\left(\alpha(\omega)-\alpha\left(\omega^{n}\right)\right)+\sum_{l} \Delta t \Gamma^{l} \Phi_{p, i j} \beta(\omega)=0$,

where $\omega$ is the physical state of block $i$ at the current timestep and $\Gamma^{l}$ is the fluid transmissibility. State-dependent operators are defined as

$$
\begin{aligned}
& \alpha(\omega)=\left(1+c_{r}\left(p-p_{r e f}\right)\right) \sum_{p=1}^{n_{p}} \rho_{p} s_{p}, \\
& \beta(\omega)=\sum_{p=1}^{n_{p}} \rho_{p}^{l} \frac{k_{r p}^{l}}{\mu_{p}^{l}} .
\end{aligned}
$$

The discretized energy conservation equation in turn can be written in operator form as

$$
\begin{aligned}
& \phi_{0} V\left[\alpha_{e f}(\omega)-\alpha_{e f}\left(\omega^{n}\right)\right]+\left(1-\phi_{0}\right) V U_{r}\left[\alpha_{e r}(\omega)-\alpha_{e r}\left(\omega^{n}\right)\right]+\sum_{l} \Delta t \Gamma^{l} \Phi_{p, i j} \beta_{e}(\omega) \\
& +\Delta t \sum_{l} \Gamma^{l}\left(T^{i}-T^{j}\right)\left[\phi_{0} \gamma_{e f}(\omega)+\left(1-\phi_{0}\right) \kappa_{r} \gamma_{e r}(\omega)\right]=0
\end{aligned}
$$

where:

$$
\begin{aligned}
& \alpha_{e f}(\omega)=\left(1+c_{r}\left(p-p_{r e f}\right)\right) \sum_{p=1}^{n_{p}} \rho_{p} s_{p} U_{p}, \\
& \alpha_{e r}(\omega)=\frac{1}{1+c_{r}\left(p-p_{r e f}\right)}, \\
& \beta_{e}(\omega)=\sum_{p=1}^{n_{p}} h_{p}^{l} \rho_{p}^{l} \frac{k_{r p}^{l}}{\mu_{p}^{l}}, \\
& \gamma_{e f}(\omega)=\left(1+c_{r}\left(p-p_{r e f}\right)\right) \sum_{p=1}^{n_{p}} s_{p} \kappa_{p}, \\
& \gamma_{e r}(\omega)=\alpha_{e r}(\omega) .
\end{aligned}
$$

This agglomeration of different physical terms into a single nonlinear operator simplifies the implementation of nonlinear solution framework. Instead of performing complex evaluations of each property and its derivatives to nonlinear unknowns, one can parameterize operators in physical space in the supporting points of mesh introduced in the physical space. The evaluation of operators during the simulation is based on multi-linear interpolation, which simplifies the linearization stage. Besides, due to the unified piece-wise representation of operators, the nonlinearity of the system is reduced, which improves the nonlinear behavior (Khait and Voskov, 2018a; 2018b).

\section{Optimal spatial discretization}

In general, high-resolution grids are necessary to capture details of fast convective flow in high-permeable regions (such as fractures (Hui et al., 2018)), while thermal conductive flow may require a lower level of grid discretization. Additionally, the computational efficiency depends on the number of degrees of freedom of the model (Faigle et al., 2015), correlating directly with the grid resolution. The first step in our study is to propose an optimal spatial discretization with enough accuracy and efficiency for modeling geothermal applications in naturally fractured reservoirs. This section describes the parameters of the selected fracture network, explains the model construction with predefined resolutions, shows the results of the numerical convergence study, and proposes the optimal grid resolution.

\subsection{Models description}

A synthetic fracture configuration is selected to perform an analysis on grid discretization and heat transfer mechanisms. A doublet for injection and production is placed in the model, see Fig. 2 for details. For simplicity of the analysis, we assume that the matrix is isotropic and homogeneous with a permeability of $0.001 \mathrm{mD}$. The aperture of the fracture is $3 \mathrm{e}-4 \mathrm{~m}$ and the cubic law (Berkowitz, 2002) is used to describe the hydraulic conductivity of the fracture. The fracture permeability with an aperture $b$ is expressed as

$k_{f}=\frac{b^{2}}{12}$.

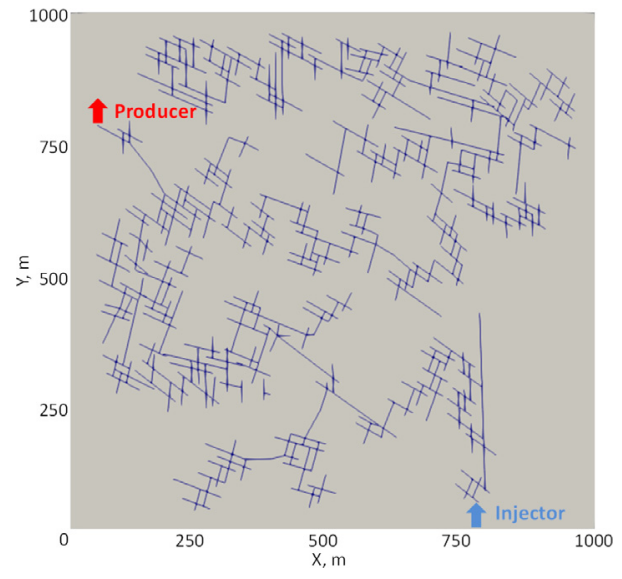

Fig. 2. The size of the synthetic fracture network is $1000 \mathrm{~m} \times 1000 \mathrm{~m} \times 50 \mathrm{~m}$. The blue lines delineate the fracture network and the solid gray represents the matrix. (For interpretation of the references to colour in this figure legend, the reader is referred to the web version of this article.)

Table 1

The number of grid cells for different grid sets.

\begin{tabular}{lll}
\hline Grid set & Fracture cells & Matrix cells \\
\hline Grid 1 & 276 & 702 \\
Grid 2 & 1,088 & 4,516 \\
Grid 3 & 1,628 & 11,954 \\
Grid 4 & 3,211 & 46,446 \\
\hline
\end{tabular}

Two different initial conditions are selected. One is with vapor and liquid coexistence from the start of the simulation. The initial pressure is 100 bars, the initial enthalpy is $1,500 \mathrm{~kJ} / \mathrm{kg}$ which corresponds to the initial temperature of $588 \mathrm{~K}$. At these conditions, the initial steam saturation is 0.47 . The injection well condition is set with flow rate control of $350 \mathrm{~m}^{3}$ /day, the production well condition is set with bottom hole pressure (BHP) control of 80 bars. This reference conditions correspond to the typical case from a hot-spot area (e.g., The Geysers (Geysers, 2019)).

Another type of conditions corresponds to an ultra-deep geothermal reservoir filled with critical water. The initial pressure is 450 bars, the initial enthalpy is $660 \mathrm{~kJ} / \mathrm{kg}$ which corresponds to the temperature of $423 \mathrm{~K}$. The injection well condition is set with flow rate control of 300 $\mathrm{m}^{3}$ /day, the production well condition is set with bottom hole pressure (BHP) control of 400 bars.

\subsection{Spatial discretization at different resolutions}

An optimal grid resolution is essential for both accurately presenting the fracture network and improving computational performance (de Hoop et al., 2019; 2020). Here, four levels of discretized models, increasing resolution from Grid 1 to 4 as shown in Fig. 3, are selected to characterize the fracture network. Table 1 lists the detailed grid numbers of different grid sets. The sensitivity of simulation results to the grid resolution will be identified with different models. To capture the fast convective flow in the fracture, the number of grid cells utilized to discretize the fractures has to stay dense across different levels of resolutions.

Comparing the numerical solutions of different discretization ratios will help to propose an optimal grid resolution for thermal flow in fractured reservoirs. The temporal variation of production temperature in conjunction with the parameter distribution (e.g., the temperature, saturation, and pressure) at the selected time is utilized to quantitatively compare the results between different models. The solutions for the grid with the highest resolution (Fig. 3d) are taken as the reference and com- 


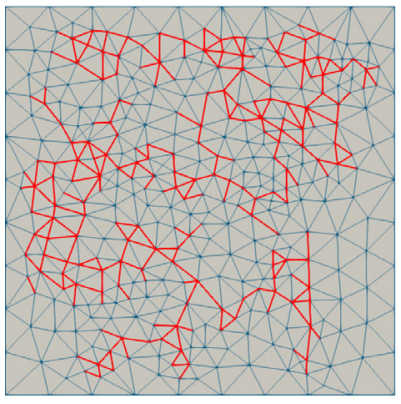

(a)

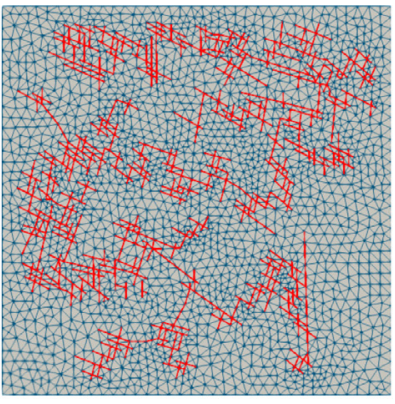

(b)

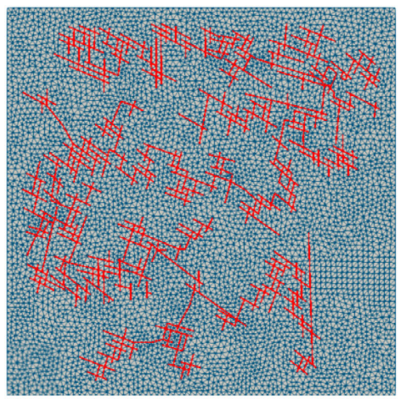

(c)

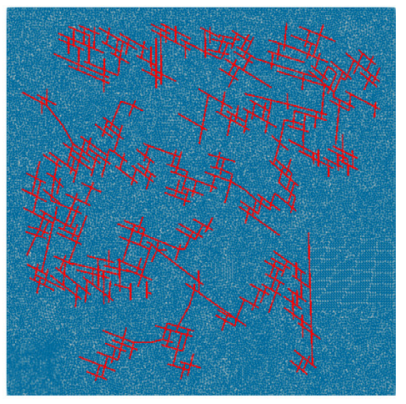

(d)

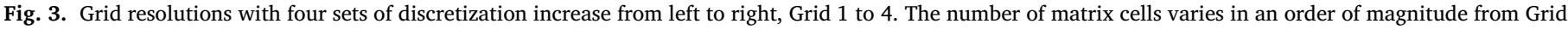

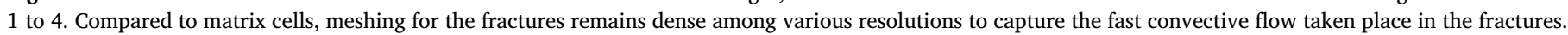

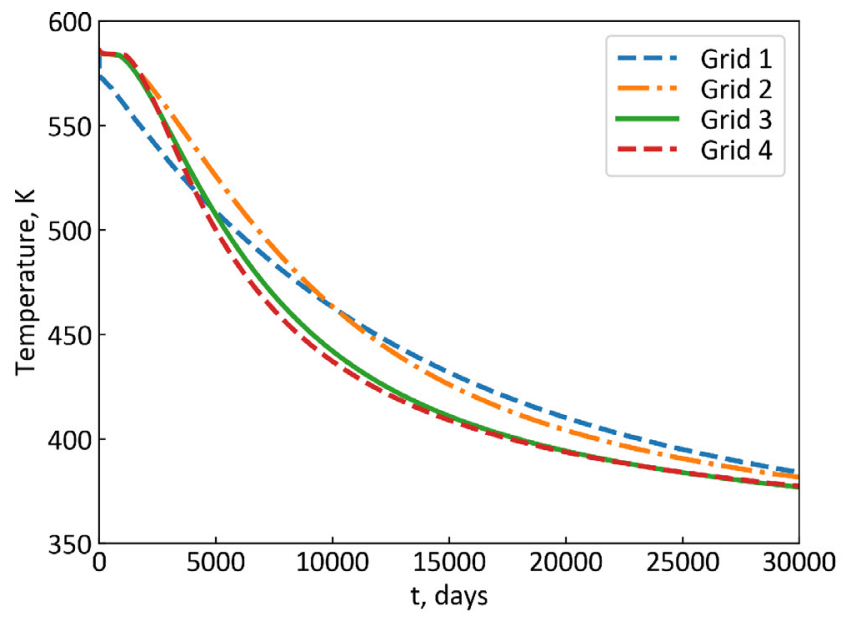

(a)

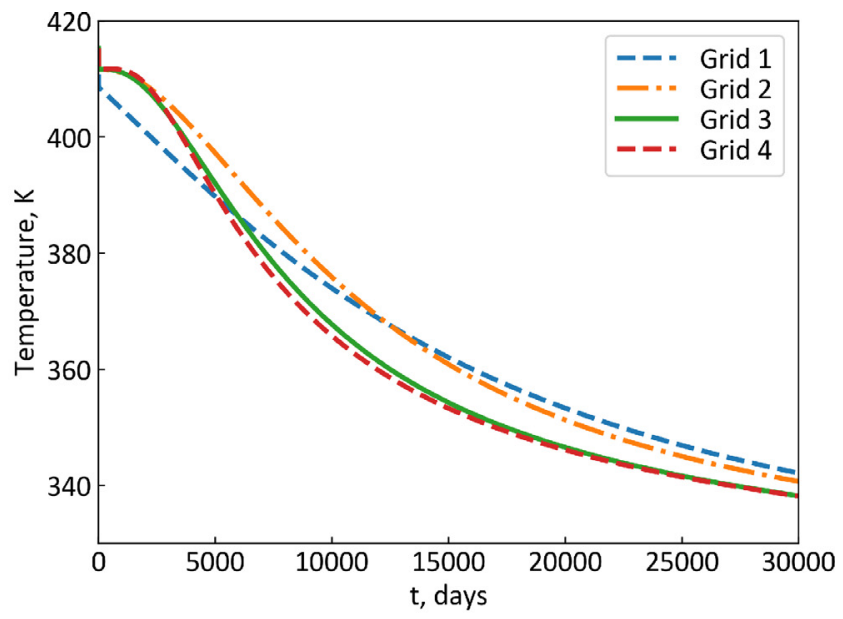

(b)

Fig. 4. Production temperature for initial conditions with (a) two phases (b) critical water with different levels of grid discretization.

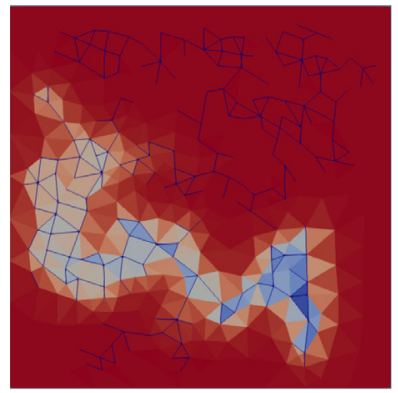

(a)

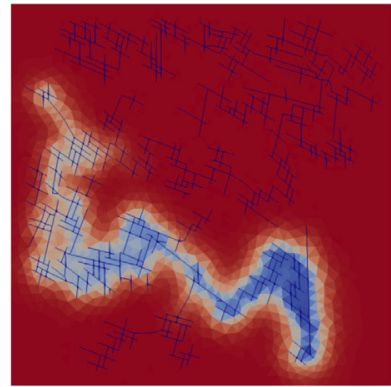

(b)

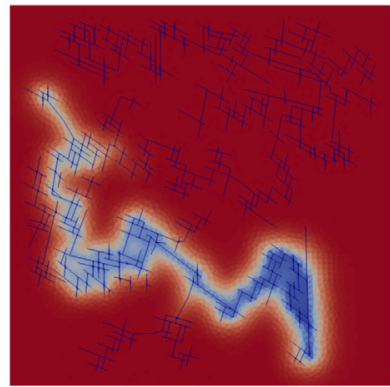

(c)

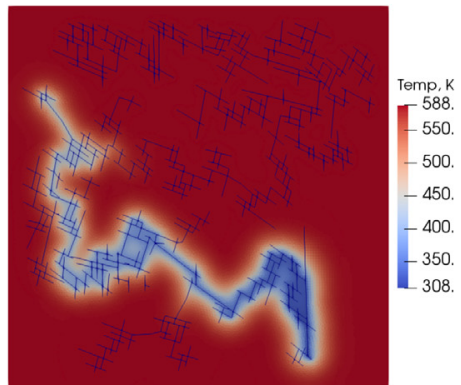

(d)

Fig. 5. The temperature maps with different levels of grid discretization at 10,000 days. The maps from left to right refer to Grid 1 to 4 .

pared with solutions generated from models at lower resolutions (Fig. 3a to c).

\subsection{Resolution study}

Direct comparison of production temperature gives evidence to the accuracy of different levels of discretization. Fig. 4 displays the temperature profile of the production well for the two test cases (two-phase and critical water conditions) under different grid resolutions. With the grid refinement, the temperature curve approaches the reference solution gradually. Grid 3 (solid green line) can closely capture the referenced temperature drop for both cases. The temperature decline (Fig. 4) can be simply divided into two consecutive parts along with time: the short rapid drop at the early period (i.e., $<7,000$ days Fig. 4a) and the fol- lowing elongated mild drop. Figs. 5 to 7 display the distribution of the temperature, pressure, and water saturation at certain selected simulation time for the two-phase system.

The temperature map for coarser resolutions (e.g., Fig. 5a) show more dispersed cold water distribution than the finer ones (e.g., Fig. 5c), which results from the assumption of instantaneous thermodynamic equilibrium within one control volume. For coarser resolutions, the averaged size of computational control volumes is larger than that under finer resolutions. For the same amount of matrix volume surrounded by several fractures, the energy depletion under a coarser grid representation will be faster than its counterpart under finer resolutions. A coarse grid block, represented by several control volumes under finer resolution, will deplete integrally when the temperature gradient exists, which enables faster energy depletion. The same amount of energy 


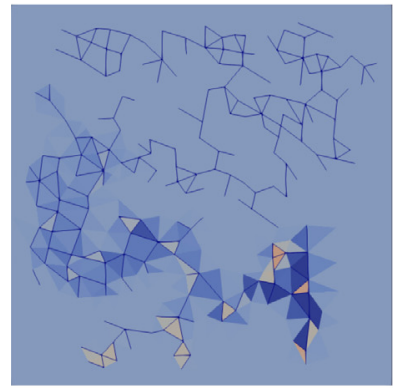

(a)

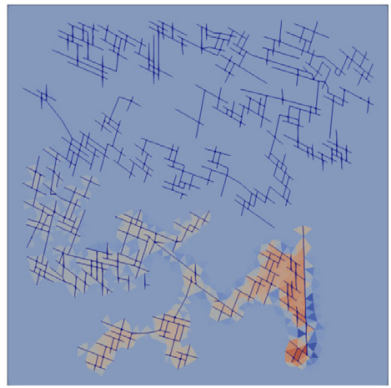

(b)

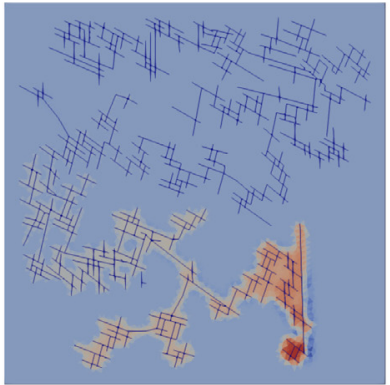

(c)

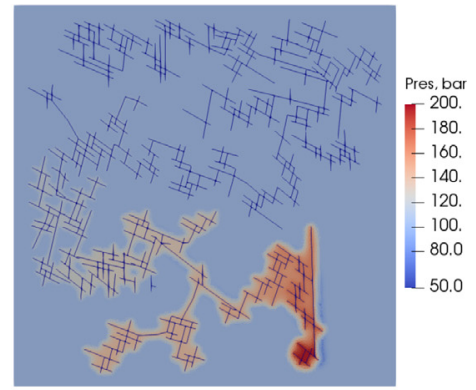

(d)

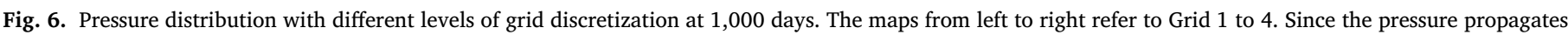
much faster than the temperature, an early simulation time (1,000 days) is selected to show the pressure distribution of the entire domain among the resolutions.

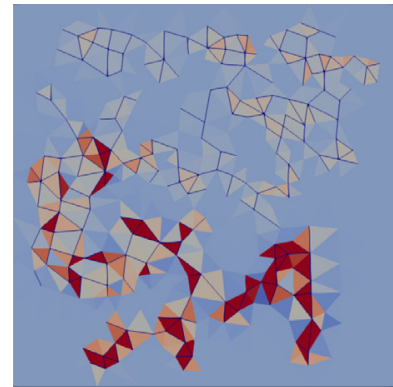

(a)

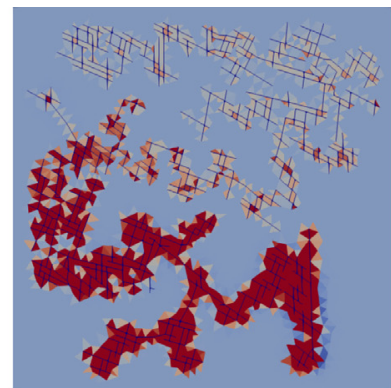

(b)

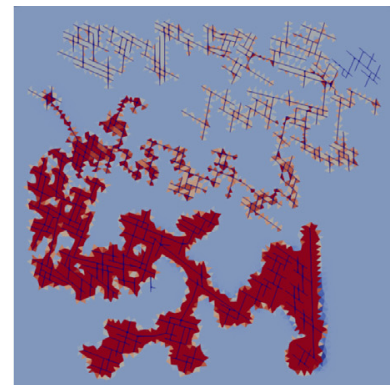

(c)

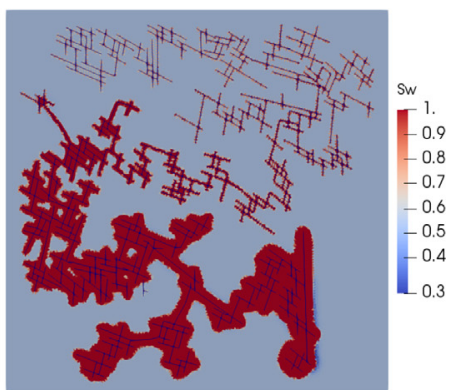

(d)

Fig. 7. The saturation distribution with different levels of grid discretization at 1,000 days. The maps from left to right refer to Grid 1 to 4 . Calculated from the solutions of pressure and enthalpy, the phase distribution at early simulation time (1,000 days) is selected.

depletion under finer resolutions will, however, experience several transitional steps between control volumes, whereby the energy depletion will slow down and conform to the referenced process. Following a similar logic, the Multiple INteracting Continua (MINC) model (Pruess and Narasimhan, 1985; Wu and Pruess, 1988) has been proposed for approximately modeling mass and heat transport in fractured systems.

Overall, the spreading of the cold water plume for different resolutions is similar and converges with refinement. The grid set 3 (Fig. 5c) can already accurately represent the solution of temperature in the computational domain. Since the initially distributed two-phase highenthalpy geothermal system is the focus of this study, the solutions of pressure and saturation also need to be checked while comparing different grid sets. It is obvious from Figs. 6 and 7 that the pressure and saturation distributions present higher sensitivity to grid resolutions than the temperature.

At the thermal front, the pore pressure will decrease subject to the steam condensation triggered by the energy depletion (Wong et al., 2018), which is a highly nonlinear process and can impact the solution of pressure in the entire domain. In comparison with the referenced pressure distribution (Fig. 6d), the pressure gradient buildup looks slower for models with coarser discretization (Fig. 6a and b). This can be explained by the larger averaged volume of computational grids, as for the temperature difference mentioned above. Saturation, as a function of enthalpy and pressure, is also sensitive to the grid resolution (Fig. 7). Restricted by the solution of pressure, the propagation of water saturation with coarser resolutions (Fig. 7a and b) cannot precisely capture the phase distribution under the reference resolution. As it is observed in (Figs. $6 \mathrm{c}$ and $7 \mathrm{c}$ ), the distributions of pressure and saturation for Grid 3 closely match with the finest resolution of Grid 4.

\subsection{Computational performance and accuracy}

The computation time of each run is measured during the simulation, as an indicator for numerical performance. Fig. 8a displays the simula-
Table 2

The nonlinear iteration for different grid sets under both critical water and two phase conditions.

\begin{tabular}{lll}
\hline Grid set & Critical water & Two phase \\
\hline Grid 1 & $3,183(0)$ & $5,147(150)$ \\
Grid 2 & $3,357(0)$ & $5,371(225)$ \\
Grid 3 & $3,575(0)$ & $5,782(480)$ \\
Grid 4 & $3,861(0)$ & $6,733(360)$ \\
\hline
\end{tabular}

tion time of both two-phase and critical water systems under various grid resolutions. The performance is scalable with the grid resolution, as an almost linear relationship is observed between the logarithm of CPU time and control volumes numbers in the serial runs. Grid 3 can run about 10 times faster than Grid 4 (the finest resolution) with reasonable accuracy as shown in Fig. 8b. It is worth noticing that the accuracy of solution depends on fractured network modification and the accuracy of nonlinear physics representation as can be seen in Fig. 8b as well. Here, the more linear critical water model converged to the reference solution faster than the two-phase model. Considering the fact that solutions at coarser resolutions (Grid 1 and 2) are not accurate, the resolution of Grid 3 is proposed for farther analysis. Besides, because of the heavier numerical nonlinearity associated with the two-phase system, more nonlinear iterations are needed than for the one with critical water, and therefore computation is more time-consuming for the twophase system. Table 2 shows the nonlinear iterations needed for both cases. The number inside the bracket represents the number of wasted nonlinear iterations.

\section{Heat and mass transfer}

Thermal convection and conduction are the two main mechanisms governing heat transfer in the subsurface. A thermal Péclet number (Pe) 


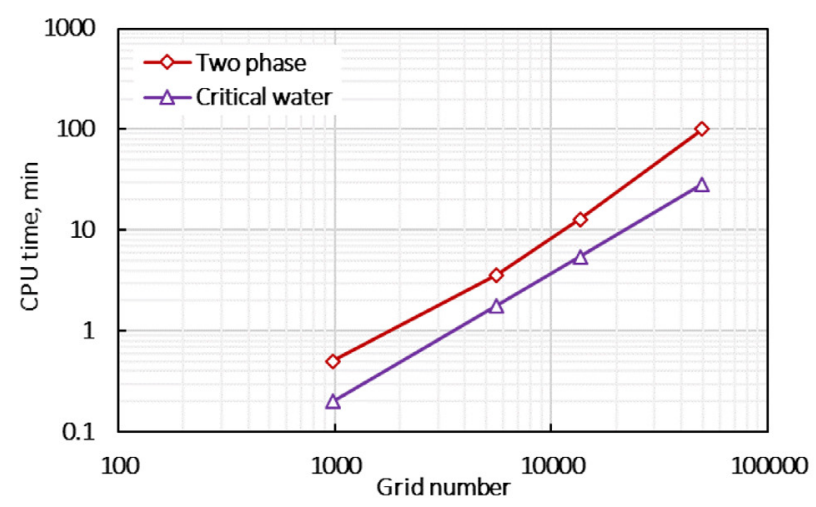

(a)

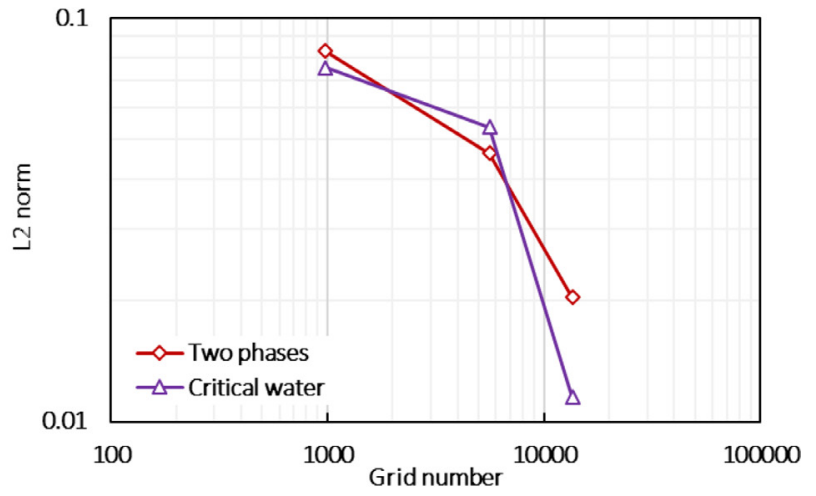

(b)

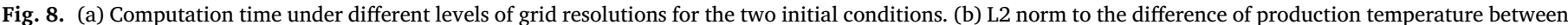
the three levels of resolutions (Grid 1 to 3 ) and referenced resolution (Grid 4).

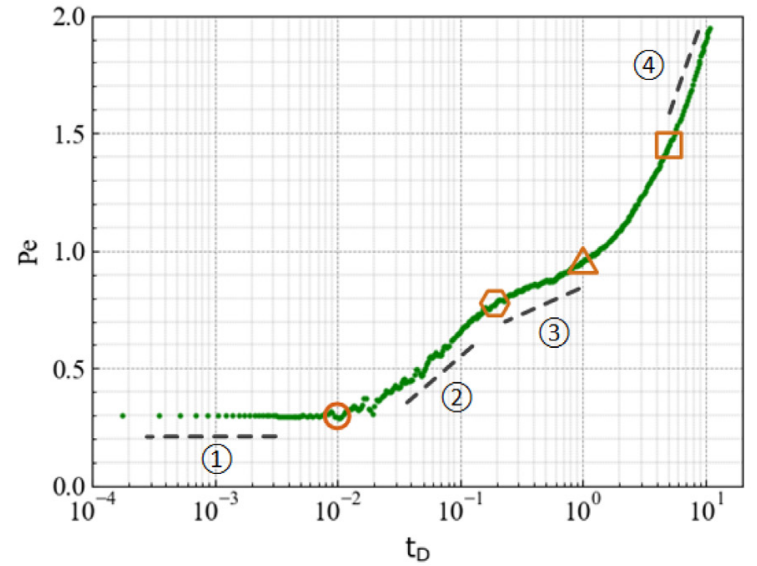

(a)
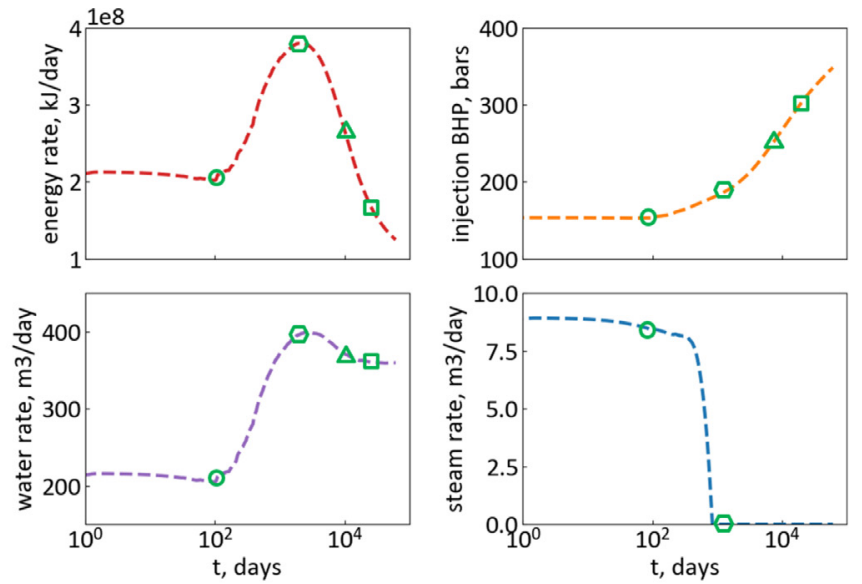

(b)

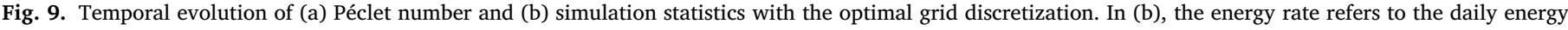

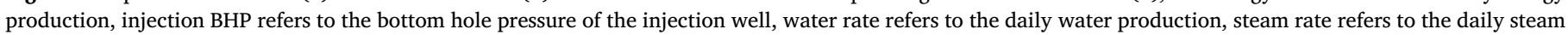
production.

is defined to quantify the interplay between thermal convection and conduction, whereby the heat transfer dynamics is studied and discussed. The optimal grid discretization (Grid 3) of the synthetic fractured reservoir is utilized in the following analysis.

\subsection{Thermal Péclet number}

For mass transfer, the Péclet number characterizes the interplay between the convective and diffusive flow. As the energy analog of mass transport, the thermal Péclet number quantifies the relative strength of convection and conduction during heat transport. The dimensionless thermal Péclet number is defined as follows:

$\mathrm{Pe}=\frac{\sum_{p=1}^{n_{p}} \int_{t} h_{p} \rho_{p} Q_{p} d t}{\sum_{i=1}^{n_{f}} \int_{t} \int_{\Omega_{\mathrm{fi}}} \operatorname{div}(\kappa \nabla T) d \Omega d t}$

where $Q_{p}$ refers to the flow rate of a specific phase ( $p=w, s$ for water and steam phase) at the production well, $\Omega_{\mathrm{fi}}$ denotes the control volumes representing the $i^{\text {th }}$ fracture. This interpretation is only suitable for fractured systems with low permeable matrix as of this study, where the thermal convective flow can be considered as dominating in the fracture network while the fracture-matrix heat exchange is through thermal conduction.
The dimensionless time is defined as follows:

$\mathrm{t}_{\mathrm{D}}=\frac{\int_{t} Q_{i} d t}{\sum_{i=1}^{n} \phi_{i} V_{i}}$

where $Q_{i}$ refers to the flow rate at the injection well, $\phi_{i}$ denotes the porosity of grid block $i, V_{i}$ denotes the volume of grid block $i, n$ denotes the number of grid blocks.

\subsection{Heat transfer regimes}

Fig. 9 a displays the temporal evolution of the Péclet number. Four stages are detected on the Péclet curve, representing different heat and mass transfer dynamics. Correspondingly, the dynamic simulation statistics for different stages are recognized in Fig. 9b. Overall, the Péclet number increases as simulation proceeds, which demonstrates the relative strength of thermal convection grows during the simulation.

\subsubsection{Stable two-phase flow}

A constant thermal Péclet number (stage (1) is observed (Fig. 9a) for the first 100 days, which indicates a stable interplay between convection and conduction. Since the energy production rate is almost constant during this period (Fig. 9b), the thermal conductive flow stays stable as well. Fig. $10 \bigcirc$ displays the temperature map of the model at 100 days. Only a 

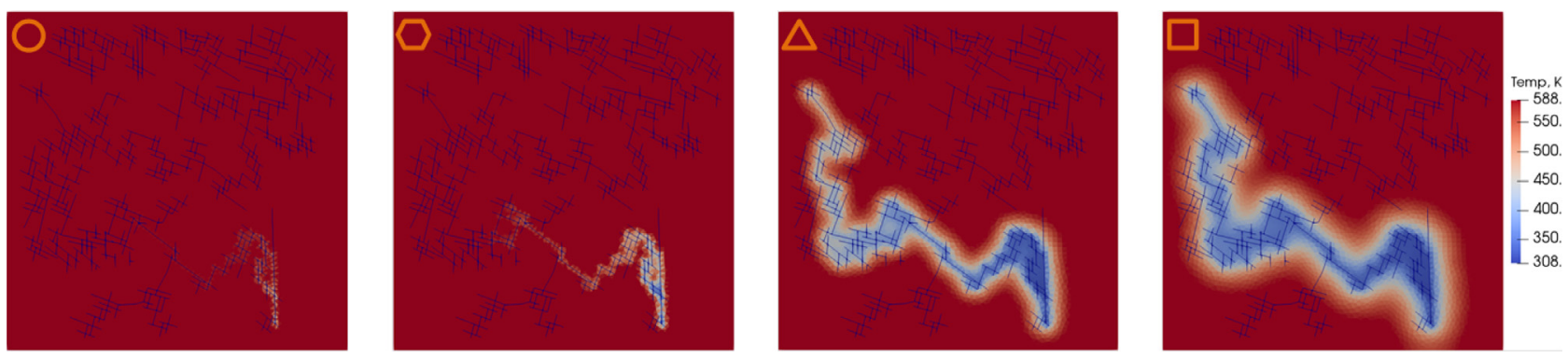

Fig. 10. Temperature propagation at four simulation time nodes: $100(\bigcirc), 2,000(\square), 10,000(\triangle)$ and $30,000(\square)$ days at fine resolution.
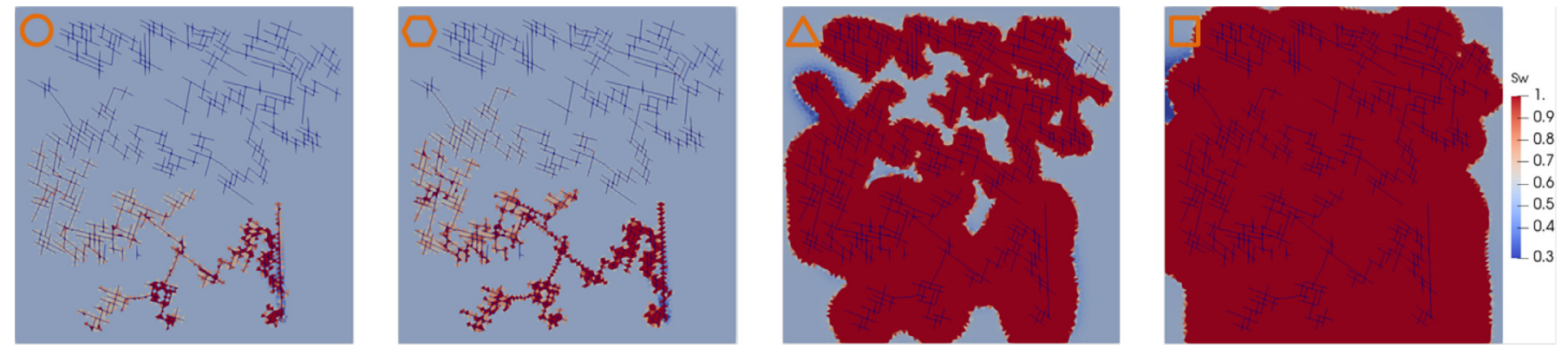

Fig. 11. Snapshot of water saturation at four simulation time nodes: $100(\bigcirc), 2,000(\square), 10,000(\triangle)$ and $30,000(\square)$ days under fine resolution.

small region of the matrix near the injection well shows a minor temperature change. As enthalpy and temperature are independent in the twophase state, the matrix temperature stays unchanged while the enthalpy drops until the two phases transit to a single phase. The phase transition (Fig. 11○) is a faster process than the temperature propagation, since

phase transition is more sensitive to pressure variations. In this period, stabilized two-phase flow occurs in the fractures, which can be deduced from the stable water and steam flow rates observed at the production well. The injected cold water, heated up by the hot rock, vaporizes in the fractures. Because of the existence of highly compressible steam, the pressure buildup within the fracture network does not take place instantaneously, which is quite different for the quasi-incompressible fluid system. Correspondingly, the needed injection pressure to sustain the operation scheme is stable (Fig. 9b).

\subsubsection{Transient two-phase flow}

As the simulation proceeds, the Péclet number increases quickly (stage (2), which mainly results from the condensed water breakthrough in fractures. As it is shown in Fig. 9b, the energy and water rates increase drastically during this period. Due to heat exploitation, the produced energy cannot support water vaporization under the fixed production pressure. Therefore, the steam rate decreases to zero and the energy production curve reaches the maximum energy rate (Fig. 9b). The water production rate increases owing to the pressure buildup in the fractured system, which can also be observed in Fig. 9b. The cold water plume penetrates deeper towards the producer (Fig. $10 \square$ ). A larger volume

near the injection well depletes by thermal conduction.

The production temperature (Fig. 4d) remains unchanged at the end of this period (2,000 days), which indicates the thermal front has not reached the production well yet. However, the fractures are fully saturated with condensed water (Fig. 9b). The saturation profile (Fig. 11 $\square$ ) shows the phase transition of the matrix cells along the fracture cells between injection and production wells at 2,000 days.

\subsubsection{Transient single-phase flow}

From 2,000 to 10,000 days, the increment of the Péclet number becomes mild (stage (3). The heat transferred by both the thermal con- vection and conduction decreases as the time proceeds. The energy contained in unit-volume of fluid drastically decreases, since the energy rate reduces sharply with just a mild decrease in water flow rate (Fig. 9b). The heat transferred by thermal conduction is expected to reduce more than by convection as the Péclet number keeps increasing during this period. From Fig. $10 \triangle$, the temperature of the matrix surrounding the

fractures decreases a lot at 10,000 days. Since thermal conduction is proportional to the temperature gradient and inversely proportional to grid distance, the thermal recharge of the fluids in the fractures by the matrix weakens along time. This can also be verified from the production temperature curve (Fig. 4a). The temperature decline in this period is sharp and steep due to the fast temperature drop in the matrix adjacent to the fractures.

As the average temperature of the fracture fluid drops, the fluid density and viscosity increase correspondingly, which leads to higher flow resistance within the fractures. The pressure needed to maintain the constant injection rate keeps increasing (Fig. 9b). Another interesting observation is the phase transition in the regions without direct contact with cold water. Owing to the pressure elevation within the model, the steam phase in the matrix condensates to water phase (Fig. $11 \triangle$ ).

\subsubsection{Stable single-phase flow}

After 10,000 days, the Péclet number rapidly increases (stage (4)). As is shown in Fig. 10 $\square$, the matrix energy has widely depleted at 30,000

days. The thermal convective flow becomes dominant as the conduction turns less influential in heat transfer due to energy extraction. The water production stabilizes in this period with only a minor decrease in the energy production rate. In parallel, the increase of injection pressure slows down simply because the temperature change of the fluids slows down after 10,000 days (Fig. 4a).

\section{Realistic fractured network}

\subsection{Background}

The fracture network used in this section is taken from an outcrop of the Whitby Mudstone Formation (Boersma et al., 2015). The hori- 


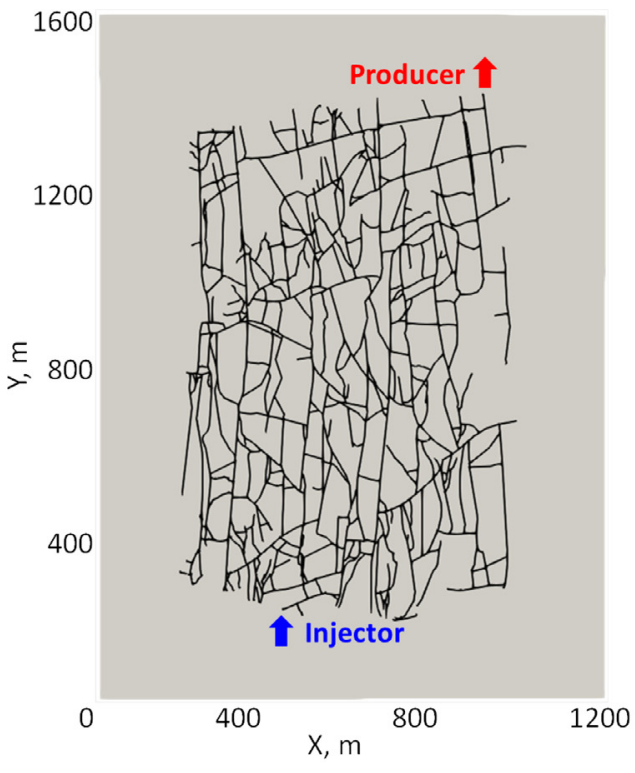

(a)
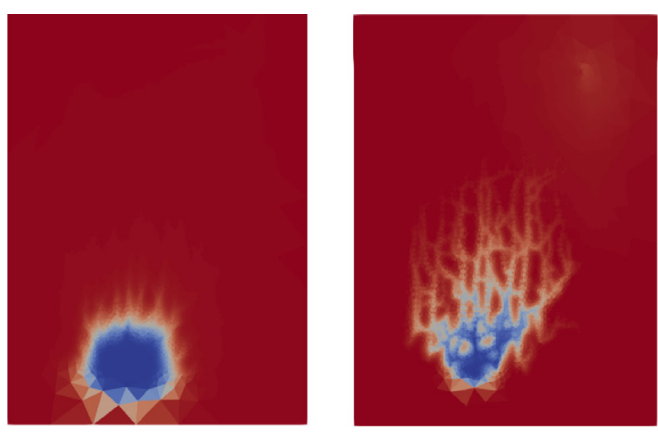

(a)

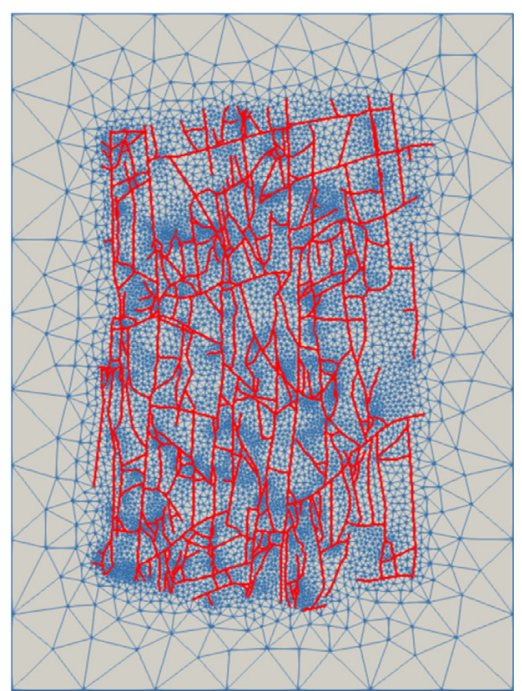

Fig. 12. The fracture network interpreted based on the bird's-eye view imagery of an outcrop at the Whitby Mudstone Formation. In (a), the black lines delineate the fracture network and the solid gray represents the matrix. (b) shows the mesh discretization with 2,234 fracture segments and 14,014 matrix cells, where the blue lines represent the mesh edges and the red lines refer to the fracture network. . (For interpretation of the references to colour in this figure legend, the reader is referred to the web version of this article.)

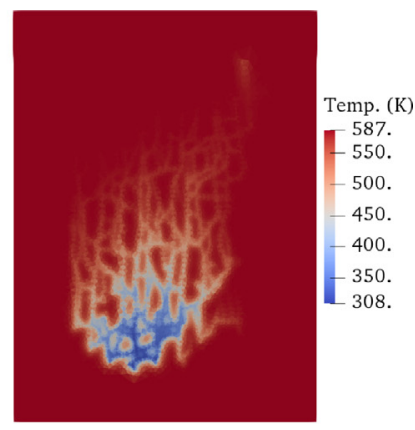

(c)
Fig. 13. The temperature distribution with different fracture-matrix permeability ratio (a) $7.5 \mathrm{e} 4$ (b) $7.5 \mathrm{e} 6$ (c) $7.5 \mathrm{e} 9$. zontal network was first captured from birds-eye view imagery of the pavement with an extent of about $15 \mathrm{~m}$ at sub-cm resolution. The imagery was interpreted in the aspect of fracture orientation, length and density. Individual fractures were manually traced from and assembled on the constructed digital map. 2,148 fracture segments were recognized from the images. The fracture network is scaled up to the size of $1200 \mathrm{~m} \times 1600 \mathrm{~m} \times 50 \mathrm{~m}$ for simulations. The geometry of the fracture network is depicted in Fig. 12a.

\subsection{Grid discretization}

A reasonable grid resolution is essential for both accurately presenting the fracture network and enhancing the computational performance. Here, a grid discretization (Fig. 12b) with characteristic length of $7.5 \mathrm{~m}$ (the same as Fig. 3c) is selected to characterize the fracture network. Compared to Wang et al. (2020a), the mesh quality is improved by the optimized treatment at the model boundary. In addition, a benchmark study against state-of-the-art research simulators has been conducted in Wang et al. (2020b).

\subsection{Numerical experiments and discussions}

In this section, we compare the heat transfer dynamics inside the model with different parameter settings: fracture-matrix permeability ratio, flow rate, rock heat conductivity and heat capacity. The parameter settings of the base case are listed in Table 3 .

\subsubsection{Fracture-matrix permeability ratio}

Permeability is one of the key factors strongly influencing thermal flow and transport. Depending on the geological formation, the matrix permeability can vary from high (porous sandstone) to low (almost impermeable basalt). The permeability contrast between fracture and matrix reflects their relative ability for fluid flow to percolate. It is important to analyze the cold front propagation under different realistic permeability ratios (Ijeje et al., 2019). A set of fracture-matrix permeability ratios is chosen and examined to observe the thermal response for different scenarios. The variation of permeability contrast is achieved by adjusting the matrix permeability, while the fracture aperture for simplicity of interpretation is fixed. Here we choose typical matrix permeability for different types of rocks: sandstone (100mD), carbonate $(1 \mathrm{mD})$ and basalt $(0.001 \mathrm{mD})$, where the fracture-matrix permeability ratio will be $7.5 \mathrm{e} 4,7.5 \mathrm{e} 6$ and $7.5 \mathrm{e} 9$.

Fig. 13 displays the temperature distribution for different permeability ratios, where large differences of the cold front propagation can be observed. For the lowest permeability contrast (Fig. 13a), the cold front propagates surrounding the injection well. Since the matrix permeability is higher in this case, the preferential heat transport along fractures is not prominent. With the increase in permeability ratio, the fluid flow predominantly occurs within the fractures instead of through the matrix, therefore the cold front spreads following the branches of the fractures towards the production well.

Fig. 14 shows the impact of fracture-matrix permeability ratio on the production temperature. With a large permeability ratio, the injected 
Table 3

Base case parameter settings.

\begin{tabular}{llll}
\hline Parameter & Value & Parameter & Value \\
\hline Matrix permeability, $\mathrm{mD}$ & 0.001 & Initial pressure, bar & 100 \\
Fracture aperture, $\mathrm{m}$ & $3 \mathrm{e}-4$ & Initial enthalpy, $\mathrm{kJ} / \mathrm{kg}$ & 1,500 \\
Fracture permeability, $\mathrm{mD}$ & $7.5 \mathrm{e} 6$ & Initial steam saturation & 0.47 \\
Rock heat conduction, $\mathrm{kJ} / \mathrm{m} / \mathrm{day} / \mathrm{K}$ & 200 & Injection well condition, $\mathrm{m}^{3} /$ day & 1,000 \\
Rock heat capacity, $\mathrm{kJ} / \mathrm{m}^{3} / \mathrm{K}$ & 2,500 & Production well condition, bar & 80 \\
\hline
\end{tabular}

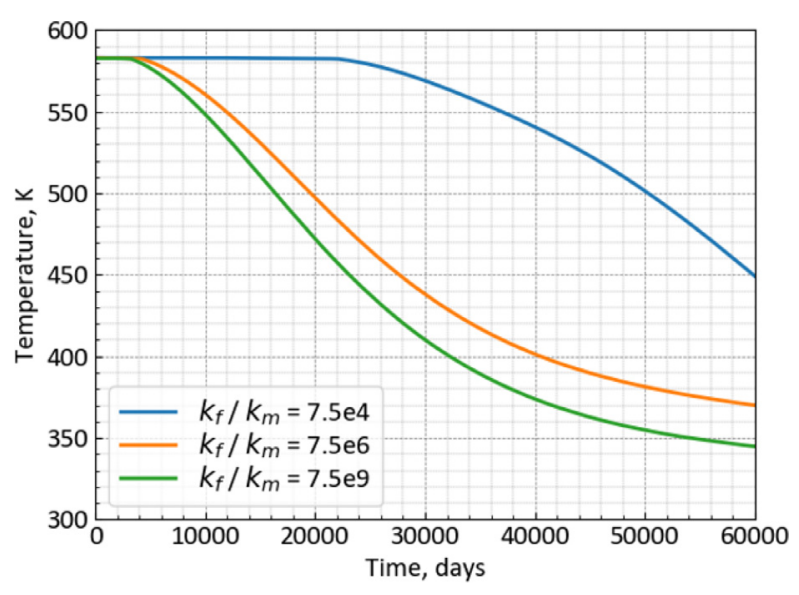

Fig. 14. Temporal evolution of the production temperature under different fracture-matrix permeability ratios.

water is pushed to flow through the fractures and the heat exchanges between fracture and matrix mainly through thermal conduction. Therefore, the amount of depleted energy is lower in fracture-dominated flow and the temperature declines faster than for lower permeability ratios. In addition, due to the diversion effect of the high-permeable matrix, fluid flow in the fractures is reduced which facilitates heating the fluids within the fractures. Consequently, the shape of the temperature curve with the lowest permeability ratio (blue line in Fig. 14) becomes largely different from the rest.

\subsubsection{Flow rate}

The flow rate directly associates with mass and heat transport. For real field applications, the flow rate is a key focus that will determine the thermal breakthrough time (Wang et al., 2019a). Therefore, it is crucial to determine how the heat transfer dynamics change with the flow rate in fractured porous media. Five different injection flow rates are utilized to investigate their influence on thermal propagation.

Fig. 15 displays the temperature distribution at different flow rates. Larger volumes deplete with the increase of flow rate. Fig. 16a shows the production temperature for different flow rates. The temperature drops earlier and faster with the elevated flow rates, resulting from the more powerful convective flow in the fractures. The cumulative energy production is displayed in Fig. 16b. Here, $500 \mathrm{~K}$ is selected as the checking point to compare the cumulative energy production with different flow rates. The amount of cumulative energy slightly increases with the reduction in flow rate, which is different from similar observations in fluvial systems (Wang et al.). The highly preferential convective flow in fractures makes the fracture-matrix heat exchange less efficient under higher flow rates. Besides, no extra flow paths get involved in heat production with the increase of flow rate, as indicated in Fig. 15.

Fig. $16 \mathrm{c}$ shows the thermal Péclet number for different flow rates. The Péclet number is constant at the early stable two-phase flow regime, where the thermal conduction is stronger than the convection under any flow rate. As the dimensionless time proceeds, the relative strength of convection exceeds that of conduction gradually. The Péclet curves overlap with each other, which means with the same amount of water injected, the relative contributions of convection and conduction to heat production are the same under different flow rates.

\subsubsection{Rock heat conduction}

Conduction plays a vital role in heat extraction during geothermal development, especially in the case of low permeable rocks. Conductive heat flow happens when the temperature gradient builds up between control volumes. The strength of thermal conduction is proportional to the magnitude of temperature gradient and heat conductivity. In this section, various rock heat conductivity values are selected within a realistic range to study the sensitivity of thermal propagation to heat conductivity in fractured reservoirs.

Fig. 17 shows the temperature distribution with different heat conductivity. With the same amount of water injected, the cold front propagation is more confined with larger heat conductivity (Fig. 17e). Since stronger conductive heat exchange happens under larger conductivity values, the reservoir energy close to the injection well largely depletes. When conductivity decreases, it will need more contact with the matrix to heat the fracture fluids and therefore, the temperature front spreads deeper towards the production well. Correspondingly, the region near the injection well weakly depletes (e.g., Fig. 17a). The influence of rock conductivity to thermal propagation is clearly nonlinear since the variations shrink as the conductivity increases.

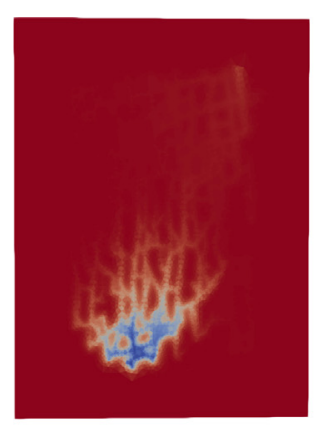

(a)

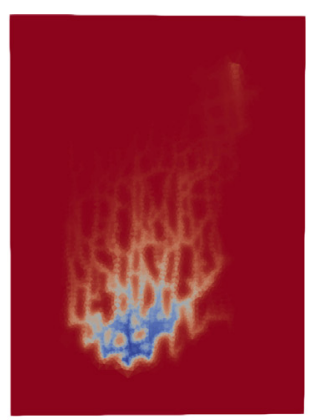

(b)

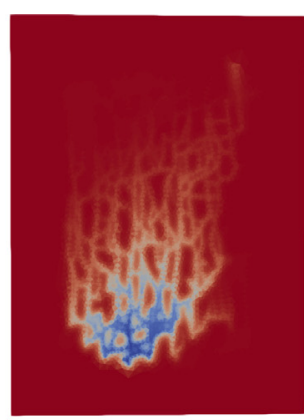

(c)

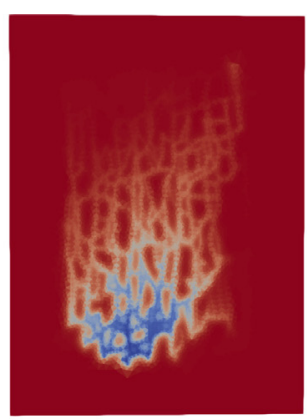

(d)

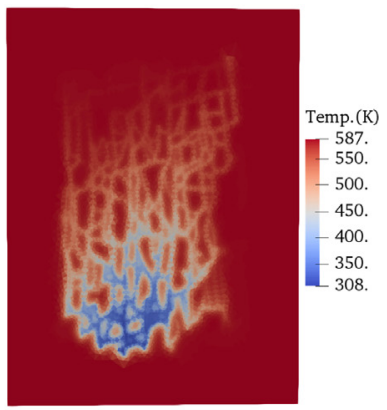

(e)

Fig. 15. The temperature distribution with different rates $\left(\mathrm{m}^{3} /\right.$ day) (a) 500 (b) 750 (c) 1000 (d) 1250 (e) 1500 . 


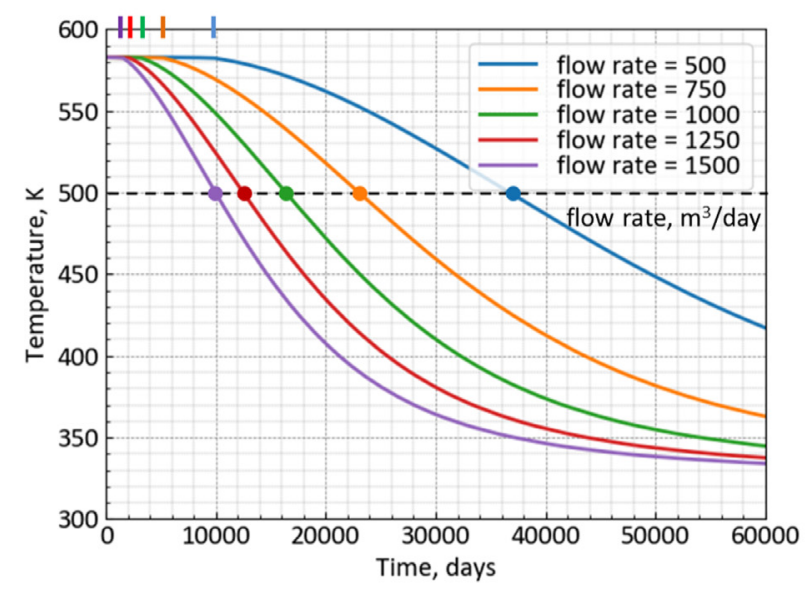

(a)

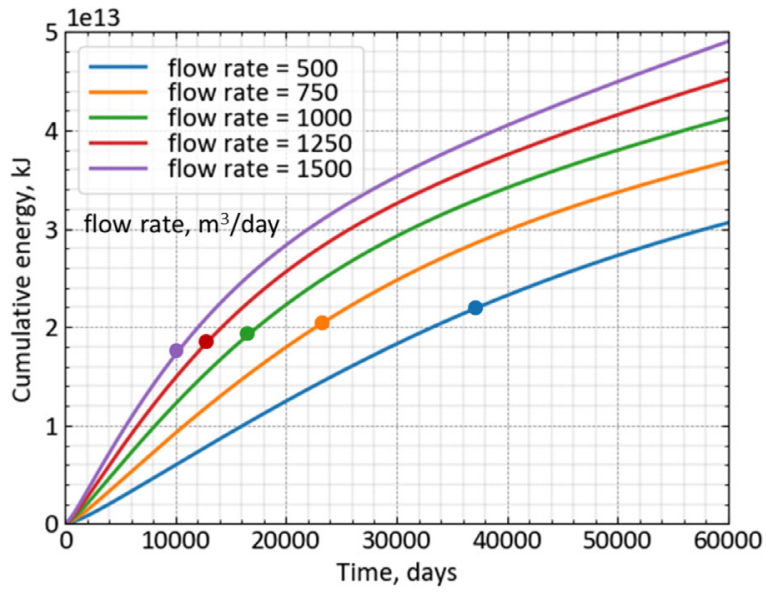

(b)

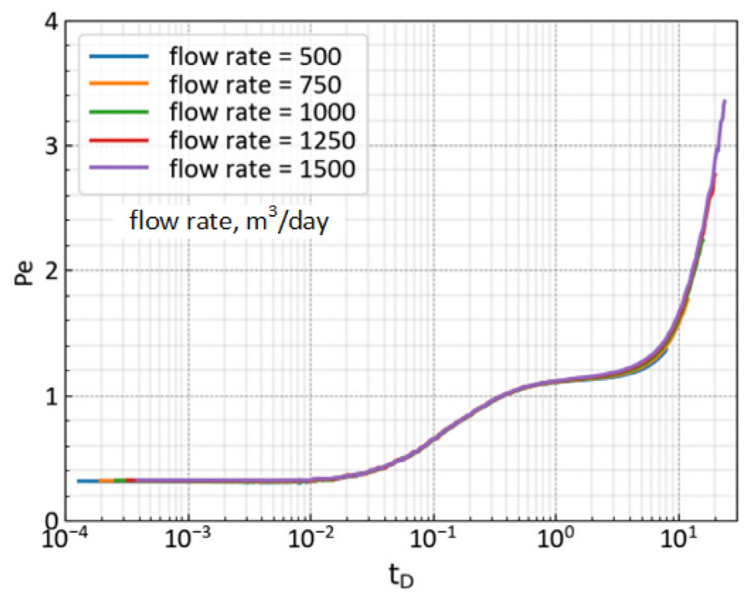

(c)

Fig. 16. Temporal evolution of (a) production temperature (b) cumulative energy production and (c) thermal Péclet number under different flow rates. The short colored lines at the top in (a) specify the time when production temperature starts dropping.

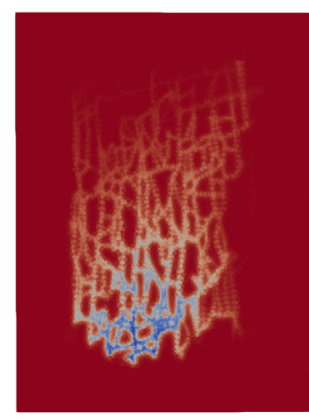

(a)

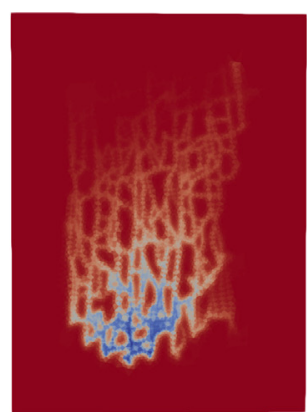

(b)

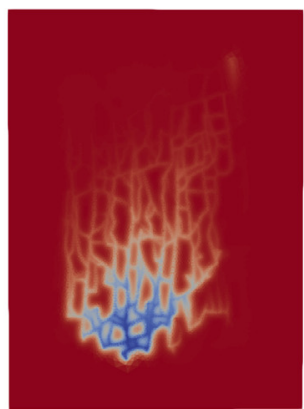

(c)

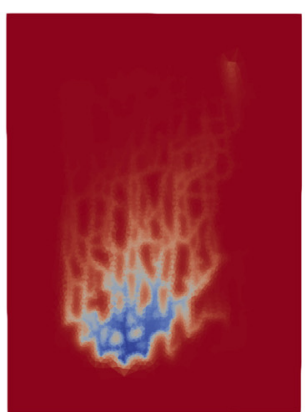

(d)

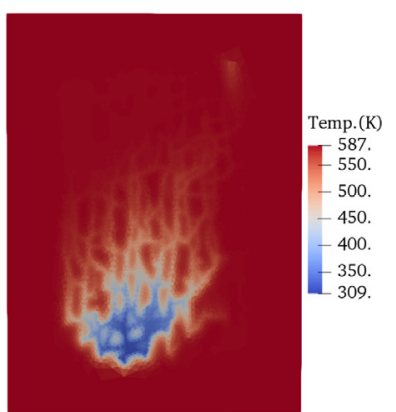

(e)

Fig. 17. Temperature distribution for realistic range of rock heat conductivity (kJ/m/day/K) (a) 50 (b) 100 (c) 200 (d) 300 (e) 400 .

The production temperature for different heat conductivity is shown in Fig. 18a. Consistent with the temperature distribution, an earlier temperature drop is observed at the production well with lower heat conductivity. The temperature decline converges as conductivity increases, as is shown for $\kappa=200,300,400 \mathrm{~kJ} / \mathrm{m} / \mathrm{day} / \mathrm{K}$, which demonstrates the conduction effect approaches its upper bound. Further increase of conductivity is unable to heat the fluids even more because either the fluid has already been heated up or the rock has been cooled down. Over- all, the variation of production temperature with conductivity is not as significant as with permeability ratio or flow rate.

The Péclet curves are similar under different heat conductivity (Fig. 18b). As a multiplier in conduction calculation, smaller heat conductivity will limit the conductive rate. However, the broader spreading of the cold front along fractures with low conductivity enlarges the contact area for heat conduction. Consequently, quite similar Péclet profiles are observed for different cases. 


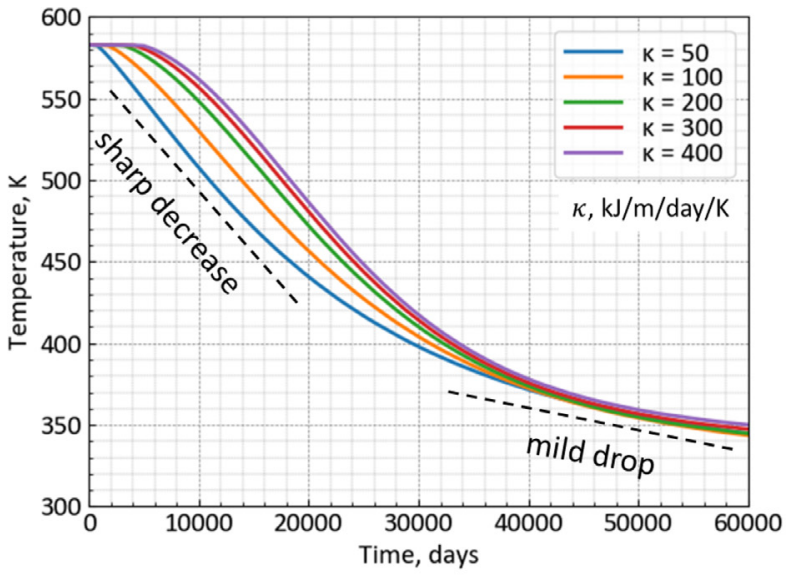

(a)

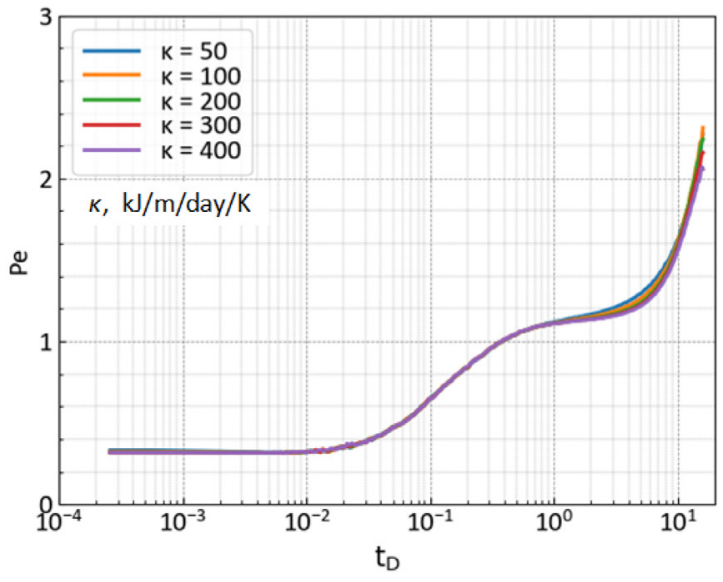

(b)

Fig. 18. Temporal evolution of (a) production temperature and (b) thermal Péclet number for realistic range of rock heat conductivity.

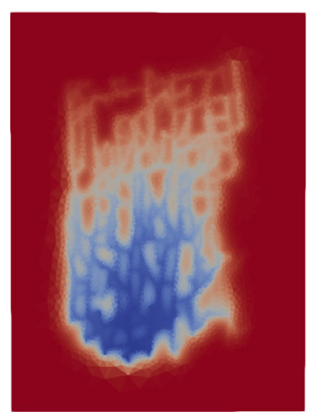

(a)

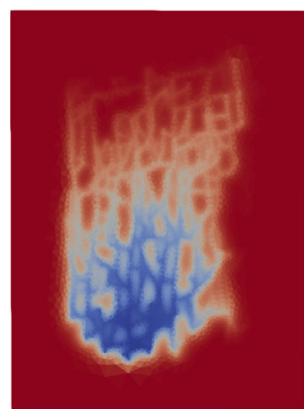

(b)

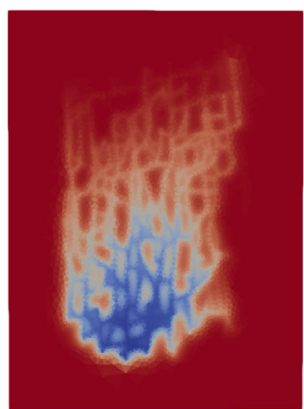

(c)

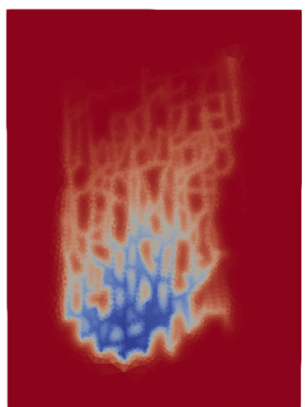

(d)

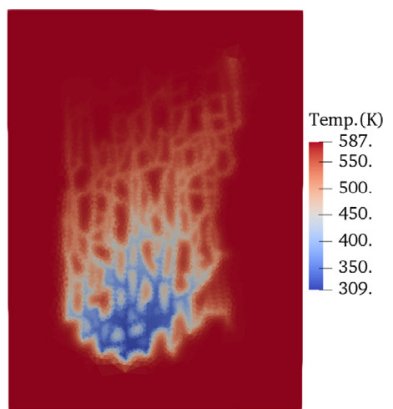

(e)

Fig. 19. Temperature distribution for realistic range of rock heat capacity $\left(\mathrm{kJ} / \mathrm{m}^{3} / \mathrm{K}\right.$ ) (a) 1500 (b) 2000 (c) 2500 (d) 3000 (e) 3500 .

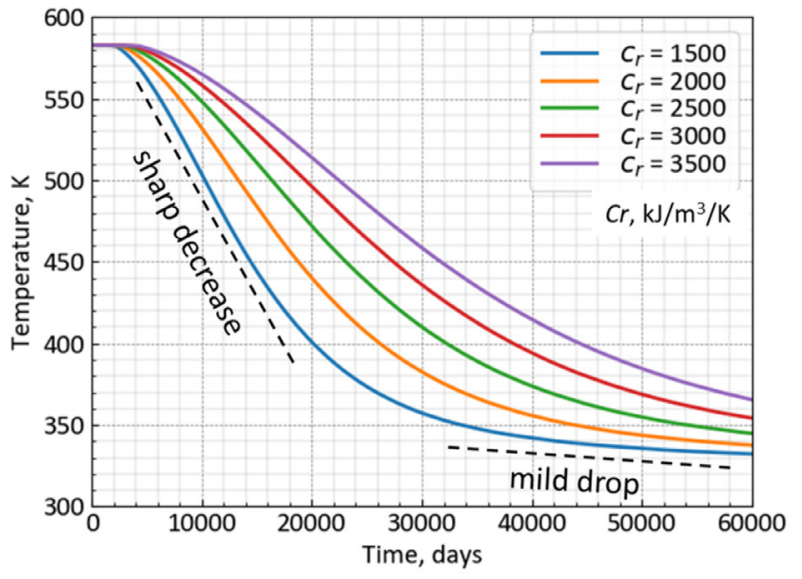

(a)

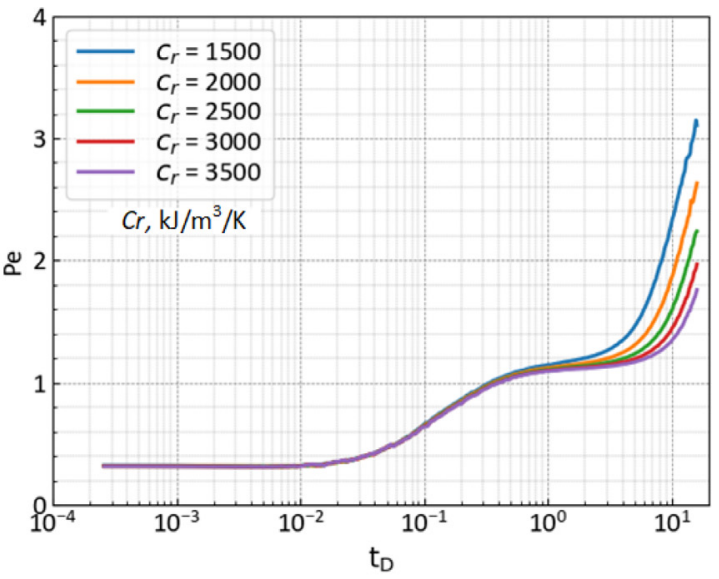

(b)

Fig. 20. Temporal evolution of (a) production temperature and (b) thermal Péclet number for realistic range of rock heat capacity.

\subsubsection{Rock heat capacity}

Volumetric heat capacity proportionally correlates with the amount of energy contained in the rock. Larger heat capacity simply means a larger amount of energy is contained in the reservoir. Here, five values of rock heat capacity within a realistic range are selected to investigate its influence on heat production.

As shown in Fig. 19, the thermal propagation is largely different from the variation in rock heat capacity, reflecting the different capabilities of reservoir rock resisting energy depletion. With the same amount of cold water injection, a larger portion of the reservoir depletes with lower heat capacity (e.g., Fig. 19a). This is because the cold water quickly depletes its bypassed reservoir via conduction and therefore, a larger reservoir volume is involved in the energy depletion process at the selected time or for certain specific simulation time.

Fig. 20 a displays the changes in production temperature with heat capacity. The production temperature shows a similar two-stage decline for all considered cases: sharp decrease and mild drop. The faster temperature drop with lower heat capacity corresponds closely with the observation in Fig. 19. Overall, the heat capacity shows a roughly linear influence on production temperature, since the difference between 
curves keeps almost the same with the linear change of heat capacity. The difference in thermal Péclet numbers (Fig. 20b) becomes noticeable as the temperature starts decreasing. Rock with larger heat capacity will supply stronger energy to the injected cold water via thermal conduction, while the convective flow can be taken as the same for different cases. Therefore, the Péclet number is smaller under larger heat capacity.

\section{Conclusion}

In this paper, the multiphase mass and heat transport in fractured reservoirs is numerically investigated using the Delft Advanced Research Terra Simulator (DARTS). Fractures are explicitly depicted with the discrete-fracture model (DFM) and the mesh quality of the DFM discretization is improved through a pre-processing procedure. Based on the numerically converged model, a sensitivity study of heat transfer in fractured reservoirs with different numerical and physical parameters is performed to guide the uncertainty quantification and optimization processes.

First, a simulation framework is presented to comprehensively investigate the sensitivity of simulation results to mesh resolution with a synthetic fractured model. The DFM discretization is based on conformal coarsening of fine-scale fractured network. The numerical convergence of the solutions is achieved with mesh refinement under highenthalpy condition with the presence of both single- and two-phase fluids. The simulation time and error statistics are summarized for different sets of mesh discretization. The optimal mesh discretization is selected out of several resolutions. It is highly recommended to qualify the mesh discretization when modeling geothermal transport within fractured systems using accurate fractured models, which will both guarantee precise simulation results and greatly improve computational performance. Subsequently, a thermal Péclet number is defined for fractured systems with low permeable matrix. Four different flow stages and relative strength between thermal convection and conduction are recognized from the Péclet curves. Our results show that geothermal development in a fractured system becomes less efficient when heat transport is mostly convection dominated, as clearly indicated by the thermal Péclet number analysis.

Next, a fracture model sketched from a direct image interpretation of a realistic outcrop is discretized with the optimal characteristic length and utilized for parameter sensitivity analysis. Almost linear impact of flow rate and rock heat capacity to production temperature is observed, whereas rock heat conductivity displays a clearly nonlinear influence which is difficult to predict without the direct numerical simulation. Additionally, the fracture-matrix permeability ratio can largely alter the propagation of cold water front. These notable impacts of investigated parameters on thermal propagation and heat transfer dynamics can help to better design and layout the system and estimate the project economics with further uncertainty analyses.

The proposed numerical methodology for finding an optimal grid resolution is of great importance for improving the computation time without compromising the accuracy. Therefore, our approach is recommended for sensitivity studies or uncertainty quantification of geothermal energy production from fractured reservoirs. In addition, the coarser resolution, though yielding less accurate solutions, demonstrates the more efficient computational performance and can be used for effective clustering of realizations in uncertainty quantification studies, see examples in (de Hoop et al., 2019; 2020). The defined Péclet number provides a way to theoretically quantify the characteristics of flow and heat transport in fractured geothermal reservoirs. Besides, utilizing a fracture network generated from real outcrop observations helps to understand the complex thermal transport in realistic fractured systems. Since there are not many publications on two-phase thermal transport in highly resolved fractured high-enthalpy geothermal system, this paper fills a gap in the literature and highlights the basic influential factors for an optimal development of such resources.
This study is performed under the assumption of constant fracture aperture. In Van Der Kooij et al. (2020), we demonstrated that the statistical variability in fracture aperture for a fixed fracture network can lead to different connectivity between injection and production wells which in turn yields different optimal production rate. In addition, rigorous consideration of the effect of thermoporoelastic deformation of rock on heat production (Stefansson et al., 2020; Berge et al., 2020) can be essential for geothermal applications and has been ongoing development in our group.

\section{Declaration of Competing Interest}

The authors declare that they have no known competing financial interests or personal relationships that could have appeared to influence the work reported in this paper.

\section{CRediT authorship contribution statement}

Yang Wang: Methodology, Software, Writing - original draft, Writing - review \& editing. Stephan de Hoop: Methodology, Software. Denis Voskov: Conceptualization, Methodology, Supervision. David Bruhn: Project administration, Funding acquisition. Giovanni Bertotti: Project administration, Funding acquisition.

\section{Acknowledgements}

We thank the financial support from China Scholarship Council.

\section{Supplementary material}

Supplementary material associated with this article can be found, in the online version, at doi:10.1016/j.advwatres.2021.103985.

\section{References}

Aravena, D., Muoz, M., Morata, D., Lahsen, A., Parada, M., Dobson, P., 2016. Assessment of high enthalpy geothermal resources and promising areas of Chile. Geothermics 59, 1-13. https://doi.org/10.1016/j.geothermics.2015.09.001.

Awadalla, T., Voskov, D., 2018. Modeling of gas flow in confined formations at different scales. Fuel 234 (4), 1354-1366. https://doi.org/10.1016/j.fuel.2018.08.008.

Berge, R., Berre, I., Keilegavlen, E., Nordbotten, J., Wohlmuth, B., 2020. Finite volume discretization for poroelastic media with fractures modeled by contact mechanics. Int J Numer Methods Eng 121 (1), 644-663. https://doi.org/10.1002/nme.6238.

Berkowitz, B., 2002. Characterizing flow and transport in fractured geological media: a review. Adv Water Resour 25 (8-12), 861-884. https://doi.org/10.1016/S0309-1708(02)00042-8.

Berre, I., Boon, W., Flemisch, B., Fumagalli, A., Glser, D., Keilegavlen, E., Scotti, A., Stefansson, I., Tatomir, A., Brenner, K., Burbulla, S., Devloo, P., Duran, O., Favino, M., Hennicker, J., Lee, I.-H., Lipnikov, K., Masson, R., Mosthaf, K., Nestola, M., Ni, C.F., Nikitin, K., Schdle, P., Svyatskiy, D., Yanbarisov, R., Zulian, P., 2021. Verification benchmarks for single-phase flow in three-dimensional fractured porous media. Adv Water Resour 147. https://doi.org/10.1016/j.advwatres.2020.103759.

Berre, I., Doster, F., Keilegavlen, E., 2019. Flow in fractured porous media: a review of conceptual models and discretization approaches. Transp Porous Media 130 (1), 215236. https://doi.org/10.1007/s11242-018-1171-6.

Boersma, Q., Hardebol, N., Houben, M., Barnhoorn, A., Drury, M., 2015. Fracture-fault network characterization of pavement imagery of the Whitby Mudstone, Yorkshire. EGU General Assembly.

Chen, S., Ding, B., Gong, L., Huang, Z., Yu, B., Sun, S., 2019. Comparison of multifield coupling numerical simulation in hot dry rock thermal exploitation of enhanced geothermal systems. Advances in Geo-Energy Research 3 (4), 396-409. https://doi.org/10.26804/ager.2019.04.07.

Coats, K., 1980. Reservoir simulation: a general model formulation and associated physi$\mathrm{cal} /$ numerical sources of instability. Boundary and Interior Layers-Computational and Asymptotic Methods.

Coats, K., George, W., Marcum, B., Chu, C., 1974. Three-dimensional stimulation of steamflooding.. Soc Pet Eng AIME J 14 (6), 573-592. https://doi.org/10.2118/4500-PA.

Collins, D., Nghiem, L., Li, Y.-K., Grabenstetter, J., 1992. An efficient approach to adaptiveimplicit compositional simulation with an equation of state. SPE Reservoir Eng 7, 259-264. https://doi.org/10.2118/15133-PA.

DARTS, 2019. Delft Advanced Research Terra Simulator.

Faigle, B., Elfeel, M., Helmig, R., Becker, B., Flemisch, B., Geiger, S., 2015. Multi-physics modeling of non-isothermal compositional flow on adaptive grids. Comput Methods Appl Mech Eng 292, 16-34. https://doi.org/10.1016/j.cma.2014.11.030.

Faust, C., Mercer, J., 1979. Geothermal reservoir simulation: 2. numerical solution techniques for liquid and vapor dominated hydrothermal systems. Water Resour Res 15, 31-46. https://doi.org/10.1029/WR015i001p00031. 
Flemisch, B., Berre, I., Boon, W., Fumagalli, A., Schwenck, N., Scotti, A., Stefansson, I., Tatomir, A., 2018. Benchmarks for single-phase flow in fractured porous media. Adv Water Resour 111, 239-258. https://doi.org/10.1016/j.advwatres.2017.10.036.

Garipov, T., Tomin, P., Rin, R., Voskov, D., Tchelepi, H., 2018. Unified thermocompositional-mechanical framework for reservoir simulation. Comput. Geosci. 22 (4), 1039-1057. https://doi.org/10.1007/s10596-018-9737-5.

Gerke, H., van Genuchten, M., 1993. A dualporosity model for simulating the preferential movement of water and solutes in structured porous media. Water Resour Res 29 (2), 305-319. https://doi.org/10.1029/92WR02339.

Gerke, H., van Genuchten, M., 1993. Evaluation of a firstorder water transfer term for variably saturated dualporosity flow models. Water Resour Res 29 (4), 1225-1238. https://doi.org/10.1029/92WR02467.

Geysers, T., 2019. The Geysers.

Glser, D., Helmig, R., Flemisch, B., Class, H., 2017. A discrete fracture model for two-phase flow in fractured porous media. Adv Water Resour 110, 335-348. https://doi.org/10.1016/j.advwatres.2017.10.031.

Hajibeygi, H., Karvounis, D., Jenny, P., 2011. A hierarchical fracture model for the iterative multiscale finite volume method. J Comput Phys 230 (24), 8729-8743. https://doi.org/10.1016/j.jcp.2011.08.021.

Hein, P., Kolditz, O., Grke, U.-J., Bucher, A., Shao, H., 2016. A numerical study on the sustainability and efficiency of borehole heat exchanger coupled ground source heat pump systems. Appl Therm Eng 100, 421-433. https://doi.org/10.1016/j.applthermaleng.2016.02.039.

de Hoop, S., Voskov, D., Bertotti, G., 2019. Uncertainty quantification and history matching for naturally fractured carbonate reservoirs. In: 3rd EAGE WIPIC Workshop: Reservoir Management in Carbonates, pp. 1-6.

de Hoop, S., Voskov, D., Bertotti, G., 2020. Studying the effects of heterogeneity on dissolution processes using operator based linearization and high resolution LiDAR data. In: 17th European Conference on the Mathematics of Oil Recovery, pp. 1-13.

Hui, M.-H., Karimi-Fard, M., Mallison, B., Durlofsky, L., 2018. A general modeling framework for simulating complex recovery processes in fractured reservoirs at different resolutions. SPE J. 23 (2), 598-613. https://doi.org/10.2118/182621-pa.

Ijeje, J., Gan, Q., Cai, J., 2019. Influence of permeability anisotropy on heat transfer and permeability evolution in geothermal reservoir. Advances in Geo-Energy Research 3 (1). https://doi.org/10.26804/ager.2019.01.03. 43-41

Jiang, J., Younis, R., 2015. A multimechanistic multicontinuum model for simulating shale gas reservoir with complex fractured system. Fuel 161, 333-344. https://doi.org/10.1016/j.fuel.2015.08.069.

Karimi-Fard, M., Durlofsky, L., 2016. A general gridding, discretization, and coarsening methodology for modeling flow in porous formations with discrete geological features. Adv Water Resour 96, 354-372. https://doi.org/10.1016/j.advwatres.2016.07.019.

Karimi-Fard, M., Durlofsky, L., Aziz, K., 2004. An efficient discrete-fracture model applicable for general-purpose reservoir simulators. SPE J. 9 (2), 227-236. https://doi.org/10.2118/88812-PA.

Khait, M., 2019. Delft advanced research terra simulator: general purpose reservoir simulator with operator-based linearization. Ph.D. Dissertation https://doi.org/10.4233/uuid:5f0f9b80-a7d6-488d-9bd2-d68b9d7b4b87.

Khait, M., Voskov, D., 2018. Adaptive parameterization for solving of thermal/compositional nonlinear flow and transport with buoyancy. SPE J. 23, 522-534. https://doi.org/10.2118/182685-PA.

Khait, M., Voskov, D., 2018. Operator-based linearization for efficient modeling of geothermal processes. Geothermics 74, 7-18. https://doi.org/10.1016/j.geothermics.2018.01.012.

Kivanc Ates, H., Serpen, U., 2016. Power plant selection for medium to high enthalpy geothermal resources of turkey. Energy 102, 287-301. https://doi.org/10.1016/j.energy.2016.02.069.

Kretzschmar, H.-J., Wagner, W., 2019. International steam tables: properties of water and steam based on the industrial formulation IAPWS-IF97. https://doi.org/10.1007/978-3-662-53219-5.

Lee, S., Jensen, C., Lough, M., 2000. Efficient finite-difference model for flow in a reservoir with multiple length-scale fractures. SPE J. 5 (3), 268-275. https://doi.org/10.2118/65095-PA.

Li, L., Voskov, D., 2021. A novel hybrid model for multiphase flow in complex multi-scale fractured systems. J PETROL SCI ENG 203, 108657. https://doi.org/10.1016/j.petrol.2021.108657, In press.

Limberger, J., Boxem, T., Pluymaekers, M., Bruhn, D., Manzella, A., Calcagno, P., Beekman, F., Cloetingh, S., van Wees, J.-D., 2018. Geothermal energy in deep aquifers: a global assessment of the resource base for direct heat utilization. Renewable Sustainable Energy Rev. 82, 961-975. https://doi.org/10.1016/j.rser.2017.09.084.

Nissen, A., Keilegavlen, E., Sandve, T., Berre, I., Nordbotten, J., 2018. Heterogeneity preserving upscaling for heat transport in fractured geothermal reservoirs. Comput. Geosci. 22 (2), 451-467. https://doi.org/10.1007/s10596-017-9704-6.
O'Sullivan, M., Yeh, A., Mannington, W., 2010. Renewability of geothermal resources. Geothermics 39 (4), 314-320. https://doi.org/10.1016/j.geothermics.2010.09.003.

Pruess, K., 1983. Heat transfer in fractured geothermal reservoirs with boiling. Water Resour Res 19 (1), 201-208. https://doi.org/10.1029/WR019i001p00201.

Pruess, K., 1990. Modeling of geothermal reservoirs: fundamental processes, computer simulation and field applications. Geothermics 19 (1), 3-15. https://doi.org/10.1016/0375-6505(90)90062-G.

Pruess, K., Calore, C., Celati, R., Wu, Y., 1987. An analytical solution for heat transfer at a boiling front moving through a porous medium. Int J Heat Mass Transf 30 (12), 2595-2602. https://doi.org/10.1016/0017-9310(87)90140-2.

Pruess, K., Narasimhan, T., 1985. A practical method for modeling fluid and heat flow in fractured porous media. SPE J. 25 (1), 14-26. https://doi.org/10.2118/10509-PA.

Pruess, K., Oldenburg, C.M., Moridis, G.J., 1999. TOUGH2 USER'S GUIDE. Technical Report.

Randolph, J., Saar, M., 2018. Combining geothermal energy capture with geologic carbon dioxide sequestration. Geophys Res Lett 38 (10). https://doi.org/10.1029/2011GL047265.

Saeid, S., Al-Khoury, R., Nick, H., Barends, F., 2014. Experimental-numerical study of heat flow in deep low-enthalpy geothermal conditions. Renew Energy 62, 716-730. https://doi.org/10.1016/j.renene.2013.08.037.

Serpen, U., Aksoy, N., ngr, T., Korkmaz, E., 2009. Geothermal energy in Turkey: 2008 update. Geothermics 38 (2), 227-237. https://doi.org/10.1016/j.geothermics.2009.01.002.

Simpson, M., Bignall, G., 2016. Undeveloped high-enthalpy geothermal fields of the taupo volcanic zone, new zealand. Geothermics 59, 325-346. https://doi.org/10.1016/j.geothermics.2015.08.006.

Stefansson, I., Berre, I., Keilegavlen, E., 2020. Finite volume discretisation of fracture deformation in thermo-poroelastic media. In: International Conference on Finite Volumes for Complex Applications, pp. 519-526.

Van Der Kooij, R., Wang, Y., Daniilidis, A., Voskov, D., 2020. Performance influences of a deep high temperature fractured geothermal system. In: 1st Geoscience and Engineering in Energy Transition Conference, pp. 1-5.

Voskov, D., 2017. Operator-based linearization approach for modeling of multiphase multi-component flow in porous media. J Comput Phys 337, 275-288. https://doi.org/10.1016/j.jcp.2017.02.041.

Voskov, D., Tchelepi, H., 2012. Comparison of nonlinear formulations for two-phase multicomponent EoS based simulation. Journal of Petroleum Science and Engineering 8283, 101-111. https://doi.org/10.1016/j.petrol.2011.10.012.

Wang, Y., de Hoop, S., Voskov, D., Bruhn, D., Bertotti, G., 2020. Modeling of high-enthalpy geothermal projects in fractured reservoirs. In: World Geothermal Congress, pp. 1-12.

Wang, Y., Khait, M., Voskov, D., Saeid, S., Bruhn, D., 2019. Benchmark test and sensitivity analysis for geothermal applications in the netherlands. In: Workshop on Geothermal Reservoir Engineering, pp. 1-11.

Wang, Y., Khait, M., Voskov, D., Saeid, S., Bruhn, D., 2019. High-enthalpy geothermal simulation with continuous localization in physics. In: Workshop on Geothermal Reservoir Engineering, pp. 1-8.

Wang, Y., Khait, M., Voskov, D., Saeid, S., Bruhn, D., under review. Influential factors to the development of low-enthalpy geothermal energy: a sensitivity study of realistic field.

Wang, Y., Li, T., Chen, Y., Ma, G., 2019. A three-dimensional thermo-hydromechanical coupled model for enhanced geothermal systems (EGS) embedded with discrete fracture networks. Comput Methods Appl Mech Eng 356, 465-489. https://doi.org/10.1016/j.cma.2019.06.037.

Wang, Y., Voskov, D., Khait, M., Bruhn, D., 2020. An efficient numerical simulator for geothermal simulation: a benchmark study. Appl Energy 264. https://doi.org/10.1016/j.apenergy.2020.114693.

Warren, J., Root, P., 1963. The behavior of naturally fractured reservoirs. SPE J. 3, 245 255. https://doi.org/10.2118/426-PA.

Willems, C., Nick, H., Weltje, G., Bruhn, D., 2017. An evaluation of interferences in heat production from low enthalpy geothermal doublets systems. Energy 135, 500-512. https://doi.org/10.1016/j.energy.2017.06.129.

Wong, Z., Horne, R., Tchelepi, H., 2018. Sequential implicit nonlinear solver for geothermal simulation. $\mathrm{J}$ Comput Phys 368, 236-253. https://doi.org/10.1016/j.jcp.2018.04.043.

Wong, Z.Y., Horne, R., Voskov, D., 2015. A geothermal reservoir simulator in AD-GPRS In: World Geothermal Congress, pp. 1-9.

Wu, Y., Pruess, K., 1988. A multiple-porosity method for simulation of naturally fractured petroleum reservoirs. SPE Reservoir Eng 3 (1), 327-336. https://doi.org/10.2118/15129-PA. 